
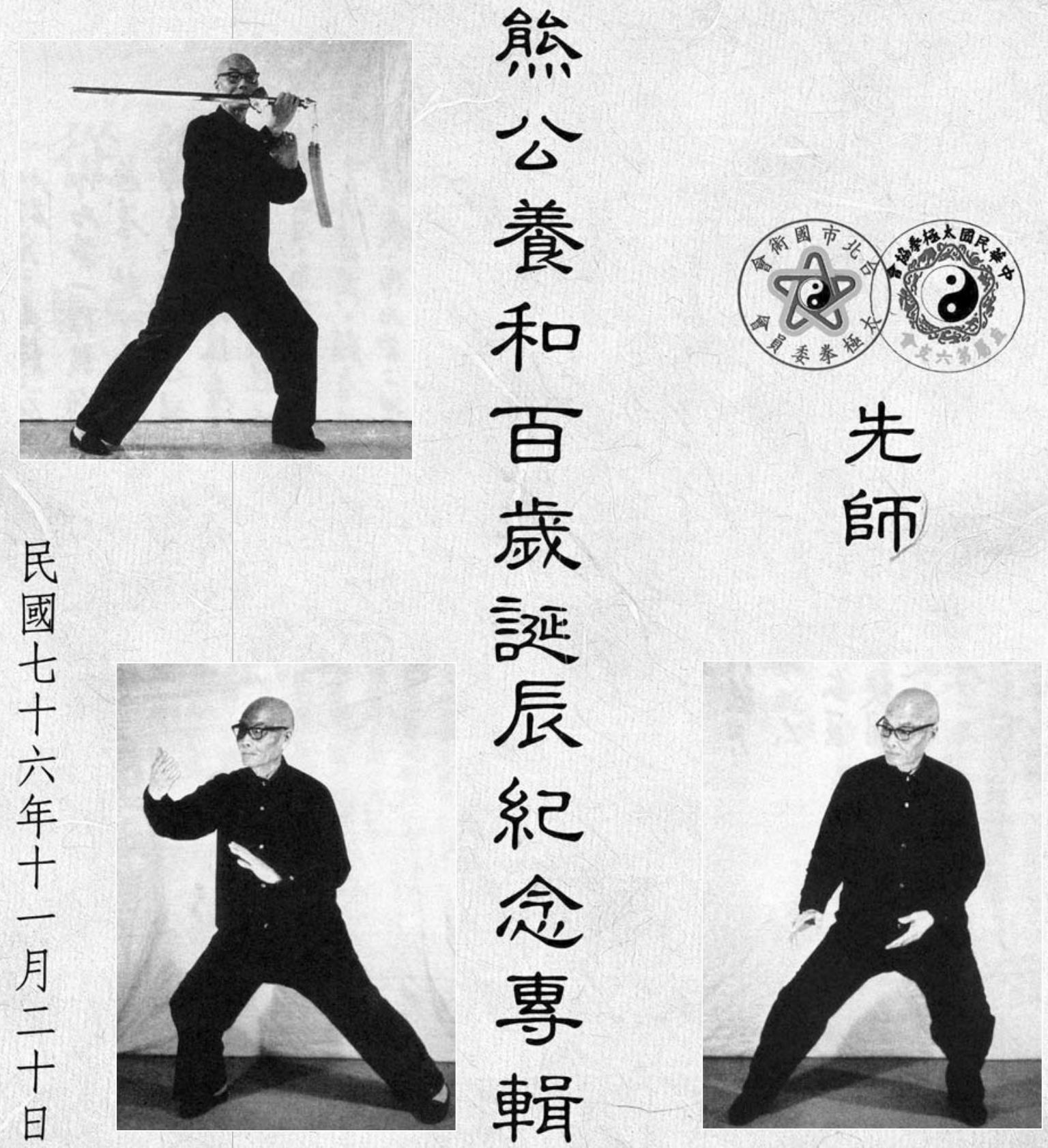

FOTOGRAFÍAS DE XIONG

YANGHE SUPERPUESTAS

EN LA PORTADA DE UNA

EDICIÓN ESPECIAL

CONMEMORATIVA

PUBLICADA CON MOTIVO

DEL CENTENARIO

DE SU NACIMIENTO

(“CONMEMORATIVO", 1987).

Colección del autor.

\section{Resumen}

Este artículo presenta una rama del taijiquan de la familia Yang poco conocida fuera de Taiwán. Se denomina estilo Xiong, debido a Xiong Yanghe (1888 1981). Sin embargo, lo poco que se ha publicado en inglés sobre el maestro Xiong es confuso y a veces contradictorio. Utilizando fuentes publicadas en chino (incluyendo los propios escritos de Xiong), documentos inéditos de sus discípulos más destacados, sitios web taiwaneses relativos a la temática, observaciones personales y documentos fotográficos, la información que aquí se ha recopilado debería proporcionar una visión completa del taiji estilo Xiong. Las fuentes en lengua inglesa se han utilizado para dibujar el marco socio-político que influyó de manera profunda en los inicios del desarrollo del taiji de la familia Yang. Las principales conclusiones afectan al linaje de Xiong, su conservación del arte y su significado para el entendimiento de todas las ramas del taijiquan como arte marcial y ejercicio enfocado hacia la salud. 


\title{
Taiji estilo Xiong en Taiwán: Desarrollo Histórico \& Exposición Fotográfica Protagonizada por el Maestro Lin Jianhong
}

\author{
Michael A. DeMARCO

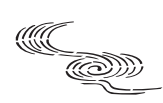

\section{Introducción}

En el denso árbol ramificado que representa el crecimiento del taijiquan a lo largo de los siglos, algunas ramas son más fuertes que otras y algunas están más altas que otras. Este artículo presenta una rama relativamente rara en la tradición de la familia Yang, asociada a Xiong Yanghe (1888 1981). Antes de profundizar en los aspectos de lo que ahora se llama estilo Xiong, primero tenemos que preguntarnos qué es lo que podemos aprender al estudiar las vidas de los principales representantes del linaje. ¿Cómo pueden influir sus teorías y prácticas de taiji sobre nuestra comprensión global del arte? Esperamos que tal investigación pueda ofrecer una mejor perspectiva histórica, a la vez que enriquece tanto nuestro entendimiento como nuestra práctica del arte.

El siguiente texto presenta aspectos relativos al linaje que juegan un papel en la formulación de una definición de taijiquan. Desde una perspectiva general de los inicios del linaje de la familia Yang, observaremos de cerca las dos ramas principales que brotaron directamente del fundador del estilo Yang, Yang Luchan (1799 1872), sus hijos y nietos, que fueron muy influyentes en el crecimiento inicial del taiji en China. Puesto que el centro de atención de este artículo es el estilo Xiong, es necesario volver la mirada hacia los profesores de Xiong y a aquellos ancestros principales que formaron el tronco principal del árbol genealógico del taiji.

En el escenario socio-político de China durante las vidas de Yang Luchan y Xiong Yanghe fueron muy comunes las invasiones extranjeras y los conflictos civiles. Este difícil periodo -deteriorado por las décadas de decadencia de la última dinastía (Qing, 1644 1911) y las siguientes décadas hasta la fundación de la República Popular de China en 1949- presenta un inmenso caudal de conocimiento que influyó en los pensamientos y acciones de los maestros de taiji anteriormente mencionados. Cada maestro tiene su propia historia. Este artículo es una breve sinopsis de la historia de Xiong Yanghe, complementada con informaciones e ilustraciones fotográficas proporcionadas por el Sr. Lin Jianhong, un instructor destacado del estilo Xiong que actualmente enseña en Taipei, Taiwán.

\section{La cuestión del linaje del taiji}

Muchos recién llegados están emocionados por comenzar el aprendizaje del taiji. Si tienen un profesor decente y un interés creciente en el arte, con el tiempo explorarán su historia, teoría y práctica. Sin embargo, pronto se ven enredados en una maraña de líneas de linaje. ¿Quién enseñó a quien? ¿Cuáles son las diferencias entre el estilo original de la familia Chen y las ramas que evolucionaron del mismo? ¿Qué enseñan realmente los principales instructores en contraste con los currículos que enseñan sus estudiantes en posteriores generaciones? Al final, ¿qué es lo que realmente aprendemos desde el entendimiento académico sobre linajes, nombres, fechas y un conjunto de historias que pueden ser verdaderas o falsas?

Fotografías cortesía

de Robert Lin-I Yu, excepto donde se indique lo contrario. 
Cuando nos acercamos a la historia del taijiquan, normalmente obtenemos una visión inicial a través de nuestro primer profesor. Esto nos introduce en el taiji. Dependiendo del profesor, el arte puede estar totalmente centrado en los aspectos educativos de la salud, adecuados para personas mayores jubiladas que buscan un modo de mantenerse en forma. Algunos profesores se concentran en el taiji como arte de combate, adecuada para guardaespaldas, militares y agentes de policía. Otros pueden enseñar ambos aspectos del arte en proporciones variables.

También hay que considerar otros aspectos si queremos comprender el taiji. Todos los profesores tienen cualidades formales y funcionales únicas: movimientos, posturas, técnicas de combate y aplicación de habilidades. Es fácil observar grandes diferencias entre los estudiantes principiantes en la mala ejecución de las formas de taiji, pero incluso los profesores del mismo linaje y generación tienen su propio toque individual, aunque puede que este sea muy sutil. Por supuesto, es importante discernir si las diferencias en los movimientos son consecuencia de una variante de la aplicación realizada de acuerdo a los principios del taiji, o si por el contrario es un movimiento incorrecto basado en un mal entendimiento de la aplicación y realizada contra los principios del taiji.

A menudo ¡cuanto más aprendemos sobre el taiji más confundidos estamos! Hay una antigua historia india que puede sernos de ayuda para tener una perspectiva de los linajes y práctica del taiji. Es la historia de seis ciegos a los que se les pidió que describieran a un elefante, sintiendo cada uno de ellos distintas partes del cuerpo del animal, tales como el colmillo, cola, tronco, pata, oreja o costado. Por supuesto, todas sus conclusiones fueron diferentes como consecuencia de sus diversas perspectivas. El "elefante" que percibían se parecía a una lanza, una pared, una serpiente, un árbol, un abanico o una cuerda. Podían discutir hasta agotarse sobre sus puntos de vista, o utilizarlos para entender mejor lo que es realmente un elefante en su totalidad.

Si buscamos realmente conocer a fondo el taiji, necesitamos ir más allá de las relativas medias verdades para lograr una perspectiva más amplia. Un estudio de los principales guardianes de cada linaje es sin duda una buena ayuda para obtener esta visión completa. En un nivel más detallado, podemos observar de cerca a los principales profesores de un linaje específico. El proceso de aprendizaje lleva muchos años, y con el tiempo veremos cómo nuestro concepto del taiji también evoluciona constantemente.

\section{Monjes ciegos examinando un elefante por Itcho Hanabusa}

ILUSTRACIÓN IMPRESA

DE UNA ESTAMPA JAPONESA (UKIYO-E)

DE LA PARÁBOLA BUDISTA MOSTRANDO A LOS MONJES CIEGOS

EXAMINANDO UN ELEFANTE. DATADA EN 1888.

Library of Congress. Call \# Illus. in H67 [Asian RR]

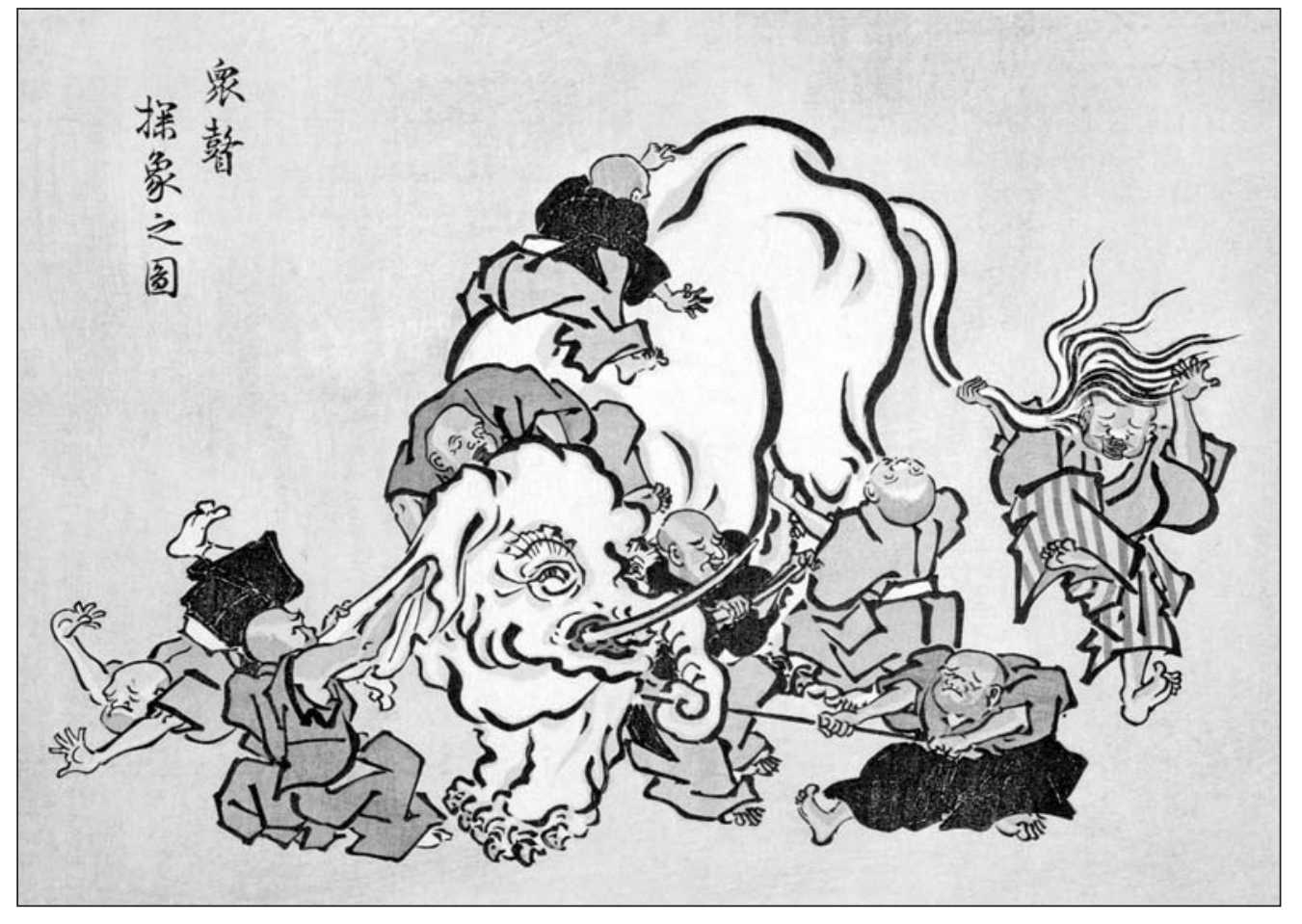




\section{Representantes del principio del linaje del estilo Yang}

Yang Luchan nació en 1799 en la comarca de Yong-

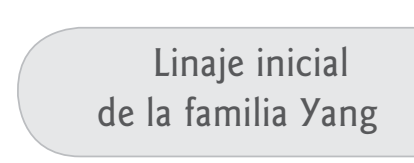
nian, provincia de Hebei. Aunque era un hombre de orígen humilde y poca cultura, amaba las artes marciales. Probablemente estudió boxeo shaolín cuando era muy joven, pero más tarde fue llevado a la aldea Chen, en la provincia de Henan, con el deseo de estudiar el taijiquan de la familia Chen. Aunque existen varias historias respecto al estudio de Luchan en la aldea Chen, los temas más frecuentes son: 1) trabajó como sirviente y estudió el taiji Chen con Chen Changxing (1771 1853), probablemente durante unos diez años, llegando a ser extraordinariamente competente en el arte, 2) regresó a su pueblo natal para enseñar a muchos el arte, y 3) se trasladó a Beijing donde fue reputado como el "Invencible Yang" y enseñó a la familia real manchú y sus guardaespaldas. Por supuesto, su estilo único de taiji llegó a ser conocido como el estilo Yang.

Sean hechos o fábulas, las historias sobre Yang Luchan no dejan duda de que poseía unas habilidades de lucha del más alto nivel. Lo que enseñó y a quién es otra cuestión. Sin duda alguna sería lógico que él hubiera continuado los antiguos precedentes y que únicamente enseñase los aspectos superiores del arte a los más cercanos.

Cuando Yang Luchan murió en 1872, dos de sus hijos continuaron la tradición del taiji de la familia. Ambos tenían un talento natural, mental y físico, para recibir la transmisión completa del conocimiento de su padre, y ambos practicaron con dedicación en un agotador régimen de entrenamiento. Los hermanos tenían personalidades muy distintas. Yang Banhou (1837 1917), el mayor, tenía un carácter a menudo descrito como duro y feroz, que manifestaba en su gusto por el combate. El hijo más joven, Yang Jianhou (1839 1917), era amable y cortés, una personalidad gracias a la cual atrajo a un gran número de estudiantes.

Aunque Yang Shaohou (1862 1930) fue el primer hijo de Yang Jianhou, Shaohou estudió principalmente con su tío, Yang Banhou. Shaohou siguió el temperamento y estilo de lucha de su tío. Ambos eran profesores duros y sólo un pequeño número de estudiantes llegaron a ser discípulos totalmente entregados. Parece que utilizaban los elementos de combate de los métodos de Yang Luchan como directriz principal de su propia práctica, que incluía la ejecución de las técnicas a máxima velocidad, saltos y patadas diversas, así como la utilización psicológica de expresiones y sonidos vocales. Como escribe Douglas Wile: "Los escritos que trazan sus orígenes hacia Yang [Banhou] son nuestro enlace más próximo a Yang [Luchan] y a la riqueza del arte antes de que se introdujese en la corriente principal de la cultura china en el siglo XX" (Wile, 1996: 93).

El segundo hijo de Yang Jianhou fue Yang Chengfu (1883 1936). Él y su hermano Shaohou enseñaron taijiquan en el Instituto de Investigación de la Cultura Física de Beijing desde 1914 hasta 1928. Fueron pioneros en llevar la instrucción al público en general. Chengfu se trasladó a Shanghai en 1928 y enseñó a muchos. Con el tiempo, el estilo particular de Chengfu se convirtió en el más extendido. Eliminó algunas de las técnicas más enérgicas de la rutina larga y enseñó a otros a practicar a un ritmo lento y constante. Aunque sin duda alguna conservaba las enseñanzas de su padre, tío y abuelo, el estilo público de Chengfu se hizo popular por sus beneficios para el mantenimiento de la salud.

Los cinco miembros de la familia Yang de los que hemos hablado en los párrafos precedentes vivieron en una época de cambios drásticos en China. Sus vidas cubren 137 años, desde el nacimiento de Yang Luchan en 1799 hasta la muerte de Yang Chengfu en 1936. Una breve visión de la historia de China durante este periodo ayudará a entender el desarrollo del taijiquan, así como el de otras artes marciales chinas que han llegado a ser populares en la época moderna. Las realidades de aquellas décadas influyeron en los modos que los primeros maestros de taiji vieron el arte, en cómo enseñaban y a quién transmitirían sus conocimientos y habilidades.

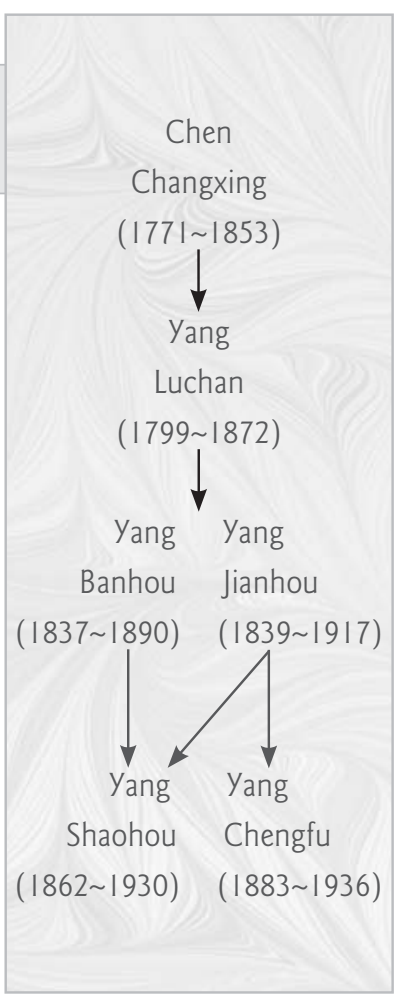

Revista de Artes Marciales Asiáticas $\diamond$ Volumen 4 Número 4 (18-39) - 2009 


\section{El efecto del espacio y del tiempo en el primer taiji estilo Yang}

¿Qué inspiró a Yang Luchan su estudio de artes marciales? ¿Fue el boxeo de la familia Chen muy diferente a otros estilos familiares desarrollados en otras aldeas? En realidad, tener a un grupo de aldeanos con un apellido común practicando boxeo en sus patios no era un fenómeno extraño durante la última mitad de la dinastía Qing (1644 1911). Philip Kuhn (1970) detalla el crecimiento de milicias locales, rebeldes, grupos de bandidos y sociedades secretas en su excelente trabajo Rebellion and Its Enemies in Late Imperial China: Militarization and Social Structure, 1796 1864 [Rebelión y sus enemigos en la China imperial tardía: militarización y estructura social, 1796 1864]. La aldea Chen es sólo un ejemplo de un pueblo que construyó sus murallas y su tradición marcial para protegerse de los ataques y robos de gente ajena, del tipo de bandidos locales.

Existen "...dos tipos básicos de instituciones militares en la sociedad china -aquellas nacidas de la prescripción estatal y aquellas nacidas de las necesidades de las unidades sociales naturales..." (Kuhn, 1970: 35). El aumento de los grupos defensivos locales llegó a ser más y más importante a medida que el gobierno Qing y sus estructuras militares y policiales se fragmentaron bajo las presiones internas y externas. Su aumento fue una respuesta directa al clima de inestabilidad socio-política.

En el siglo XVIII existía un descontento interno en toda China mientras la población aumentaba hasta el extremo en que la producción de alimentos no podía mantener el ritmo. "El crecimiento de la población sobrepasó inevitablemente la creciente producción de alimentos, y el nivel de vida comenzó a caer. La propagación de la corrupción y la indolencia en el gobierno agravó las condiciones" (Hucker, 1975: 302). Más allá del sentimiento anti-extranjero de los chinos por los gobernantes manchúes que conquistaron y gobernaron China desde 1644 a 1911, la población en general sentía que el gobierno había perdido el Mandato Divino y era incapaz de gobernar. Las sublevaciones llegaron a ser endémicas, estallando en grandes agitaciones sociales tales como la Rebelión de la Sociedad del Loto Blanco (1793 1804). Incluso más devastadora fue la Rebelión Taiping (1851 1864), en la que cerca de treinta millones de personas perdieron la vida, trayendo la destrucción a quince provincias (Wakeman, 1977: 156). A mediados del siglo XIX, en algunas provincias "dos tercios de la población fue dada por muerta o perdida" (Wakeman, 1977: 155).

Durante siglos, China se había creído el estado más civilizado del mundo. Sin embargo, las rebeliones, hambrunas e inundaciones tuvieron un gran efecto sobre el gobierno y la sociedad durante el siglo XIX. La imagen que China tenía de sí misma cambió gradualmente. No volvió a ser un país caracterizado por la riqueza y fortaleza, y los países extranjeros se aprovecharon de esta fragilidad. Con la intrusión de los mercaderes y misioneros europeos, China sintió rápidamente su debilidad respecto a las formas modernas de la guerra y de los negocios internacionales. Durante décadas, los portugueses, holandeses, británicos, franceses, americanos, rusos y japoneses aplicaron más presión sobre China mientras trataban de obtener ganancias mediante tratados desiguales, la adquisición de ciudades portuarias, el tráfico de opio y la extracción con sifones de las menguantes reservas de plata china. Las potencias extranjeras se aprovecharon de una China que se había debilitado desde el interior.

De forma paralela a la larga lista de rebeliones internas se produjeron diversas guerras con países extranjeros, tales como la Guerra del Opio (1839 1842), la Guerra Anglo-China (1856 1860), y la Guerra Chino-Francesa (1884 1885). Las usurpaciones extranjeras fueron destructivas, pero su significado real se produjo mediante los tratados resultantes, que eran desiguales en el sentido que daban una gran ventaja a las potencias extranjeras y tenían un alto coste para los chinos. La Guerra Chino-Japonesa (1884 1895) produjo una "profunda conmoción psicológica", ya que "hizo, más que ninguna otra crisis, que los chinos se viesen forzados a evaluar sus propias fortalezas y debilidades" (Wakeman, 1977: 192). Sobre todo, cada tratado humilló a los chinos, y muchos comenzaron a buscar soluciones para resolver los problemas causados por décadas de conflictos internos e influencia extranjera.

Taiji Estilo Xiong en Taiwán $\diamond$ Michael A. DeMarco 
Durante la última mitad del siglo XIX, muchos líderes políticos e intelectuales estaban enganchados en la discusión de las formas mediante las que se podría restaurar el sistema imperial Qing o un nuevo sistema político a través del "auto-fortalecimiento". Parece que la mayoría de los intentos fallaron o agravaron los problemas. Por ejemplo, una idea fue utilizar el resentimiento contra la expansión imperialista para estimular la Rebelión Bóxer (1900 1901) contra las embajadas extranjeras. Esta iniciativa estaba condenada al fracaso. "Miles de jóvenes comenzaron a practicar los ejercicios estilizados del boxeo Shaolín y [Bagua] -ejercicios que se suponía que liberaban su [qi] (pneuma) y les dotaban de una fuerza tan impresionante que repelía las balas extranjeras" (Wakeman, 1977: 217). Incluso un general militar chino "simplemente ridiculizó sus afirmaciones de invulnerabilidad contra las armas de fuego", y "puso a 50 bóxers de la Sociedad del Cinturón Dorado para comprobarlo, alineándoles contra una pared y disparándoles" (Wakeman, 1977: 218). El líder bóxer fue arrestado y decapitado. La derrota bóxer trajo nuevas imposiciones sobre los chinos y provocó incluso una mayor pérdida de poder.

A medida que el gobierno de Beijing perdía el control de las provincias, se produjo un consecuente crecimiento de poder en las zonas locales, a menudo asociado a las propias provincias. Frederic Wakeman señala que "los gobernadores provinciales de principios del siglo XX asumieron cada vez más las funciones militares y fiscales que una vez habían pertenecido al gobierno central" (Wakeman, 1977: 232). Casi en la mitad de las provincias chinas "los militares se convirtieron inmediatamente en gobernadores después [de la Revolución Wuhan (1911)], o siguientes dos o tres meses. Además, las tropas en las distintas provincias eran reclutadas en su mayor parte dentro de las provincias en las que servían; su fidelidad era claramente provincial y personal, así que los líderes militares provinciales tenían, en efecto, ejércitos personales a su disposición" (Sheridan, 1977: 147).

Mientras los ejércitos privados crecían -algunos pequeños y otros grandes- la guerra aumentaba. "Entre 1916 y 1928, la lucha entre los militaristas independientes -señores de la guerra- rompió China en fragmentos, y la maquinaria política oficial de la república que había sucedido a la monarquía -parlamento, ministros, etc.- llegó a ser en gran medida irrelevante en las realidades de la vida política china. Al mando de sus ejércitos personales, los señores de la guerra dominaban distritos, provincias y regiones, y luchaban con los generales vecinos por más territorio e ingresos" (Sheridan, 1977: 20).

La guerra fue endémica durante el periodo de los Señores de la Guerra (1916 1928). Un escritor ha -contado más de 400 guerras civiles grandes y pequeñas solo en la provincia de Szechwan- (Sheridan, 1977: 88). Con tal caos territorial, ¿cómo era posible reintegrar China bajo un gobierno nacional moderno y unificado? Cada facción de un señor de la guerra funcionaba de acuerdo a sus propios intereses y objetivos políticos. Un aspecto interesante a mencionar es cómo se entrenaba a los soldados. El decoro variaba mucho dependiendo del grupo. Algunos señores de la guerra exigían que sus tropas estuvieran muy entrenadas, a la vez que actuaban con suma compasión hacia la población en general. Su honorable código de disciplina enfatizaba el buen trato hacia todos y la contención de los vicios asociados a los soldados de pobre carácter. El general Feng Yuxiang (1882 1948), por ejemplo, "exigía una condición física extraordinaria, y sometía a sus oficiales y hombres a un entrenamiento constante y riguroso para conseguirlo. ... Prohibía la bebida, el juego, la visita a prostitutas, incluso los juramentos" (Sheridan, 1977: 74). En el otro extremo del espectro estaban otros señores de la guerra y sus tropas, que bebían alcohol, violaban y robaban a su antojo.

En medio del dinámico flujo político y militar del periodo de los señores de la guerra, la lealtad cambiaba a menudo entre ellos, así como sus oficiales y tropas (Sheridan, 1977: 58). Unas pocas facciones crecieron fuertes mientras muchas se volvieron débiles y se desintegraron o fueron absorbidas. Finalmente, dos partidos políticos principales com-

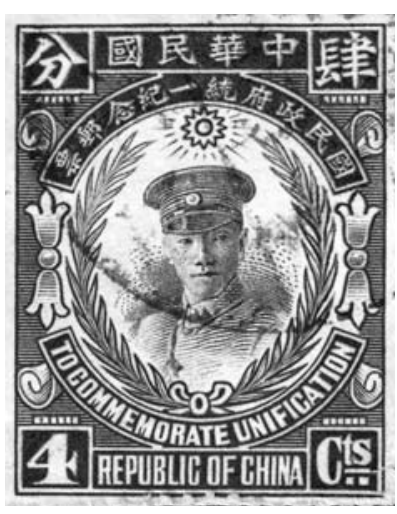

SELLO EMITIDO POR LA REPÚBLICA DE CHINA "PARA CONMEMORAR LA UNIFICACIÓN", CON EL RETRATO DEL GENERALÍSIMO CHIANG KAI-SHEK.

Cortesía de iStockphoto.com. 
pitieron por la supremacía: los Nacionalistas (Guomintang), bajo el liderazgo del general Chiang Kai-shek (1887 1975), y el Partido Comunista Chino, dirigido por Mao Zedong (1893 1976). Al principio ambos trabajaron juntos para acabar con el periodo de los señores de la guerra y expulsar a los japoneses, pero sus diferentes ideologías políticas condujeron a una inevitable guerra civil (1927 1949).

Chiang Kai-shek, encumbrado durante el periodo de los señores de la guerra, había aprendido a "centrar toda su política sobre el concepto de fuerza Había crecido durante una época de traición y violencia. ... Hubo pocos estándares de decencia humana que sus contemporáneos, señores de la guerra, no violaran. No obedecían a ninguna ley, solo a la fuerza..." (Schurmann \& Schell, 1967: 236). Mao también afrontó esta dura realidad, y su a menudo citada frase es: "El poder político crece del cañón de un arma". Chiang y Mao lucharon hasta que los Comunistas surgieron victoriosos en 1949.

Una "prolongada transformación revolucionaria" que duró más de un siglo, "pero en muchos aspectos el periodo crítico fueron los 37 años, 1912 1949, desde la caída de la monarquía y la fundación de una república hasta el establecimiento de la República Popular de China por los Comunistas. Durante este periodo republicano, la desintegración y el desorden estuvieron en su máximo" (Sheridan, 1977: 4). Todos los primeros maestros de taijiquan del estilo Yang vivieron durante esta transformación revolucionaria, y existen algunos factores comunes en sus vidas que influyeron sobre sus enseñanzas.

El factor individual más importante en el desarrollo del taijiquan fue su asociación con la defensa. Hubo siglos de bandolerismo, rebeliones a pequeña y gran escala, y actividades de sociedades secretas por toda China. Especialmente, las zonas rurales carecían de protección por el ejército o policía nacional, así que el entrenamiento en artes marciales fue utilizado para la defensa regional y local. La aldea Chen, aunque sea famosa, es sólo un ejemplo de cómo un arte marcial de familia evolucionó dentro de un pueblo amurallado. Es el lugar del estilo de taijiquan de la familia Chen original, admirado por ser un sistema de boxeo de gran calidad. Los representantes de la familia Chen, tales como Chen Changxing (1771 1853) y Chen Gengyun (1799 1872), fueron empleados como guardaespaldas de élite y personal de seguridad en el transporte de mercancías; Chen Yenxi (1848 1929) entrenó al hijo del primer presidente republicano Yuan Shikai, y también era el guardaespaldas familiar del oficial-académico Du Youmei en Boai, Henan. El hijo de Du, Du Yuzi (1886 1990), se convirtió en discípulo de Chen Yenxi.

El miedo es un gran motivador. La gente tenía que proteger sus provisiones de comida así como sus vidas. Muchos entrenaban con constancia y rigor. También tenían miedo de un cambio en las lealtades, por lo que eran cautelosos sobre a quién enseñaban. Normalmente los vínculos eran personales (enseñando a los miembros de la familia y del pueblo) y, bajo circunstancias que implicasen a una zona más amplia, los lazos serían provinciales, donde el dialecto común y las costumbres sociales reforzaban algunos vínculos afectivos.

Las décadas de grandes cambios sociales hicieron que las relaciones cambiasen. El estilo Chen se trasladó fuera de su pueblo natal, y otros, como Yang Luchan, vinieron a aprender el taiji Chen. Luchan y su hijo Nahou enseñaron a los guardias imperiales de los manchúes y a las tropas de guarnición. Algunas enseñanzas se realizaban en privado y otras en público. "Cuando se pregunta por qué los estudiantes de [Guangping] de la familia Yang exhibían técnicas tanto blandas como duras en su estilo, mientras que los estudiantes de [Beijing] mostraban sólo las técnicas blandas, [Banhou] respondía que los estudiantes de [Beijing] eran principalmente aristócratas adinerados, y que, después de todo, había una diferencia entre los chinos y los manchúes, implicando una política de resistencia pasiva a la dinastía extranjera al impartir únicamente la mitad de la transmisión del taijiquan" (Wile, 1983: ix).

Cuando nació Yang Chengfu, en 1883, su abuelo Luchan ya había muerto hacía once años, y su tío Banhou murió cuando Chengfu tenía nueve. Como resultado, el entrenamiento de Chengfu fue algo diferente del de su hermano Yang Shaohou. Shaohou y 
Banhou fueron célebres por su rudo boxeo. Internacionalmente, el estilo de Shaohou no es tan conocido como el de Yang Chengfu. Las dificultades que rodearon su vida le condujeron a suicidarse en 1930 (Yun, 2006: 55).

Las vidas de los maestros del taiji del estilo Chen y de los primeros maestros del estilo Yang son un reflejo de esa época. Las principales figuras estaban muy implicadas en la defensa a nivel local, provincial y a veces nacional. La necesidad de poseer verdaderas y sumamente efectivas habilidades marciales estaba arraigada en la conciencia de todos los que encaraban luchas a vida o muerte en sus vidas cotidianas. Conocidos profesores de artes marciales, como Yang Luchan, se vieron en el dilema de tener que decidir entre el deseo de alejar su más alto conocimiento de los "extranjeros" y el deseo de ayudar a los miembros familiares y amigos más cercanos. Y también tenían que ganarse la vida durante los tiempos difíciles.

También existió otra motivación para la enseñanza que a menudo se pasa por alto. Tiene que ver con las muchas décadas de trato humillante sufrido a manos de los países extranjeros que forzaron a China a regalar concesiones mientras perdía su propio territorio, riqueza y dignidad. Los chinos llegaron a conocerse como los "hombres enfermos de Asia". Esa frase aparece en la película de Bruce Lee Fist of Fury [Furia Oriental] (1971), y la película de Jet Li Fearless [Sin Miedo] (2006). La idea de enseñar artes marciales para la salud encaja bien con el movimiento de "auto-fortalecimiento" de principios del siglo XX. El país necesitaba hacerse fuerte, como hacía su gente.

\section{Xiong Yanghe y sus contribuciones únicas al taijiquan}

Bastante pronto, Yang Luchan y sus hijos exhibieron modos distintos de instrucción. Tenían un conjunto de estudiantes: miembros de la familia, oficiales militares, guardias manchúes, otros instructores marciales, personas ricas y campesinos. Cada uno mantenía la tradición de la familia Yang y podía adecuar su instrucción en función de la relación profesor-estudiante.

Las personalidades también jugaron su papel en los métodos de enseñanza así como en la selección de los estudiantes. ¡Había caracteres polares yin-yang entre Yang Banhou (yang) y Yang Jianhou (yin), y en la siguiente generación entre Yang Shaohou (yang) y Yang Chengfu (yin)! El estilo de Shaohou era exigente física y mentalmente, y además no se andaba con paños calientes con sus estudiantes. El estilo de Yang Chengfu llegó a ser el más popular debido a su carácter y métodos de enseñanza más agradables. El ritmo más lento y las modificaciones que hizo eran adecuados para una mayor población, como la población anciana. Su enseñanza tuvo un gran impacto sobre el "auto-fortalecimiento" nacional, trayendo salud a miles de personas.

¿Cuál es el lugar de Xiong Yanghe en este desarrollo? Dentro del linaje del taiji Yang, llevó sus enseñanzas a Taiwán siguiendo el éxodo de los Nacionalistas chinos a Taiwán en 1949 y se convirtió en la principal influencia en la expansión del taijiquan por toda la isla. Cuando murió, en 1981, él y sus discípulos más antiguos habían dado clases a más de diez mil personas. Su estilo continúa extendiéndose a través de sus discípulos, y su sistema único ahora se refiere como taiji estilo Xiong. La historia de Xiong es interesante por el lugar especial que ocupa en el taiji así como por su propia vida personal.

Xiong nació el 29 de septiembre de 1888 en la provincia de Jiangsu, comarca de Funing. Su padre, Xiong Weizhen, aprobó el examen provincial (grado militar de juren) a finales de la dinastía Qing. Yanghe estudió artes marciales primero con su padre, luego este contrató instructores para su joven hijo: a la edad de 12 años, un maestro de Shaolín llamado Liu He y su discípulo Liu Zhongfang vinieron a enseñarle; a la edad de 15, el maestro Yin Wanbang en el sistema de boxeo Jiangnan de las Ocho Armonías. Estos recibieron influencia marcial de Gan Fengchi. Cuando Xiong tenía 20 años, Tang Dianqing "Mano Milagrosa" (1850 1926), fue contratado para darle clases. Estos profesores dieron a Xiong una base excelente en boxeo shaolín y pudieron haber proporcionado a Xiong su primer contacto con el taijiquan.

LA PROVINCIA DE JIANGSU

ESTÁ EN LA COSTA ESTE DE

CHINA. XIONG YANGHE

NACIÓ EN FUNING, QUE

ESTÁ EN EL MUNICIPIO DE

YANCHENG.

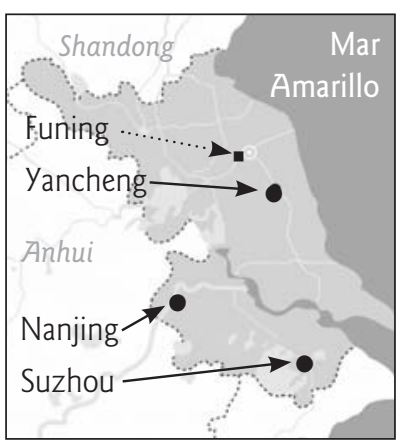

Revista de Artes Marciales Asiáticas $\diamond$ Volumen 4 Número 4 (18-39) - 2009 


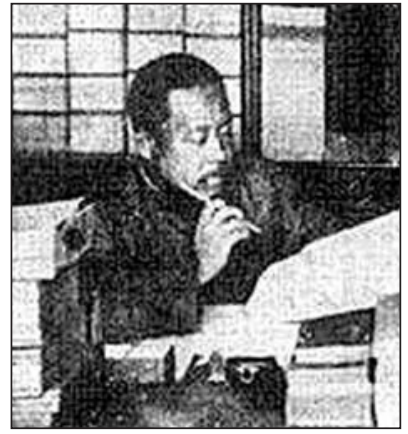

HU PUAN (1878 1947)

EN SU ESCRITORIO;

ERA FAMOSO COMO UN ERUDITO Y MAESTRO DE

TAIJIQUAN. www.taiji.net.cn

Xiong tenía experiencia práctica en el combate ya que había ayudado a su padre a mantener la seguridad de la ciudad. Se había encontrado demasiado a menudo peleando con gángsters. Cuando tenía 19 años, era campeón local de boxeo en las competiciones "sin reglas" realizadas en plataformas elevadas (leitai), como se ve en la película Fearless. Debido a la fuerza de sus patadas, Xiong se ganó el sobrenombre de "Piernas de Funing". Tales experiencias le dieron una nueva perspectiva del boxeo, pero estaba destinado a enriquecer sus artes marciales con los contactos realizados a través de su trabajo.

Cuando Xiong tenía 23 años, comenzó su carrera militar, teniendo que ver con operaciones militares y de seguridad. A los 29, era director adjunto de la oficina gubernamental de la provincia de Anhui, y a los 35 pasó a una posición de administración en las guarniciones de la comarca de Funing. Durante este periodo, Xiong coincidió con Hu Puan (1878 1947), maestro de taijiquan del estilo Yang de la vieja estructura, quien se convirtió en su profesor más influyente. El sobrenombre de Hu era "Hu Hu", que significa "El tigre Hu".

Hu había nacido en la provincia de Anhui, comarca de Jing. Sirvió como jefe de departamento de la administración civil de la provincia de Jiangsu. Como un sinólogo bien conocido por sus libros y poesía, Hu dio clases en la Universidad de Shanghai. Mientras estuvo en Shanghai, tuvo la oportunidad de conocer y estudiar con varios maestros de taiji de gran calibre. Practicó diariamente a las 6:00 de la mañana durante más de 18 años, hasta que sufrió una minusvalía a consecuencia de un derrame que le produjo una parálisis ${ }^{1}$.

¿Quién fue el principal profesor de Hu? Las fuentes difieren, señalando que estudió con:

1) Chen Weiming (1881 1958) ${ }^{2}$

2) Yang Jianhou (1839 1917) ${ }^{3}$

3) Yang Chengfu (1883 1936) ${ }^{4}$

4) Yang Shaohou $(1862 \sim 1930)^{5}$

5) Le Huanzhi (1899 1960) ${ }^{6}$

La mayoría de declaraciones respecto a los profesores de Hu simplemente dicen que estudió con tal o cual persona en particular. Además, existen dudas sobre el tiempo que Hu estudió con sus profesores. ¿Qué pudo haber aprendido de ellos? Una referencia dice que "Yang Chengfu y el discípulo Sr. Hu Puan... comparaban apuntes, realizando un estudio minucioso del taijiquan, consiguiendo perspectivas exhaustivas y perspicaces del gongfu taiji" . Afirmar que Hu era el "discípulo" de Yang Chengfu es una declaración fuerte. Desafortunadamente, no he encontrado evidencias sólidas para confirmar tal afirmación.

Probablemente, Hu Puan conoció a todos estos profesores y pudo haber estudiado con cada uno de ellos en diferentes grados. Pero es interesante señalar que hablaba extremadamente bien de Le Huanzhi (1899 1960), que era de la comarca de Gushi, en Henan. Le era médico y también un antiguo discípulo de Dong Yingjie (1898 1961). En su autobiografía publicada, Hu escribió que el taijiquan de Le es extremadamente bueno. Hu escribió que -desde su propia experiencia en el empuje de manos con Yang Chengfu, Sun Lutang, Wu Jianquan y Le Huanzhi-, Le demostró ser superior, y su toque era muy efectivo ya que tenía una fuente indetectable, "como nubes que pasan y agua que fluye", "como sin tener sustancia".

Debido a que las fuentes son confusas, es difícil saber de quién había recibido Hu Puan su instrucción en taijiquan. Además, ni siquiera se ha definido uniformemente el linaje de Xiong. Existen unas pocas fuentes que dicen que Xiong fue discípulo de Yang Shaohou'. Esto parece ser una suposición basada principalmente en lo que enseñaba Xiong. Sin embargo, tanto Xiong como Hu Puan probablemente tuvieron algún contacto con Yang Shaohou. Lo que sabemos con certeza es que, en su autobiografía (1962), Xiong sólo menciona a Hu Puan en relación con la transmisión de la vieja estructura del estilo

Taiji Estilo Xiong en Taiwán $\diamond$ Michael A. DeMarco 
Yang. Esto no niega la posibilidad de que Xiong conociera a otros maestros de taiji del estilo Yang, o que aprendiese sus métodos por medio de Hu Puan.

Xiong pudo haber "estudiado realmente con Hu Puan", pero sin duda tuvo buenas relaciones con otros maestros de taijiquan ${ }^{10}$. Una fuente señala que Xiong tuvo la oportunidad de encontrarse con Yang Jianhou mientras estaba en Beijing por negocios oficiales. Esto le dio la oportunidad de buscar consejo sobre el taijiquan, especialmente en relación con la rutina de dos personas llamada sanshou ("manos dispersantes"). En esta época, Xiong estudiaba incondicionalmente y era capaz de comprender los misterios más profundos del $\operatorname{arte}^{11}$. Aunque, otra fuente menciona que Yang Jianhou enseñaba en la comarca de Funing, y Xiong buscó su consejo para la práctica del sanshou ${ }^{12}$. Liang Dongcai (también conocido como T.T. Liang) afirma que Yang Jianhou dio clases de sanshou a Xiong. Liang también mantiene que probablemente nadie pudo haberlo aprendido de Yang Chengfu, porque su padre Yang Jianhou murió antes de que pudiera enseñárselo a él (Hayward, 2000: 61).

Puesto que Xiong tenía que tomar parte en las discusiones políticas que caían dentro del alcance de sus funciones militares oficiales, tenía grandes oportunidades para conocer a mucha gente hábil en diversas tradiciones marciales. Podían comparar sus estudios y beneficiarse observando el amplio abanico de artes marciales chinas. Durante décadas, Xiong recibió una base sólida en shaolín del norte y del sur y en el taijiquan de sus profesores personales y del contacto con otros gracias a su carrera militar. Aquí aparecen algunos de los hechos destacables de su carrera ${ }^{13}$.

\section{$\underline{\text { Edad }}$ \\ Posición}

39 años

40 años

Comandante de regimiento, Ejército Revolucionario

ctor de la agencia de seguridad pública de la comarca de Funing en la provincia de Jiangsu, y posición concurrente como director de la brigada de producción

49 años Magistrado de la comarca de Funing, provincia de Jiangsu

52 años Comandante general de brigada de seguridad

53 años Comandante asistente de seguridad

54 años Comandante mayor general de seguridad

58 años Comandante mayor general de grupo

60 años Oficial delegado comandante, Comandancia Militar

En 1949, el Partido Nacionalista, bajo las órdenes de Chiang Kai-shek, se retiró a Taiwán, y el partido Comunista estableció la República Popular de China (RPC) en el continente. Xiong dimitió y se trasladó a Taiwán cuando "aproximadamente 600.000 soldados nacionalistas y las personas a su cargo se retiraban del continente a Taiwán" ${ }^{14}$. Suele decirse que parte de esta ola migratoria incluyó a cuatro famosos "Grandes Perros" del taijiquan: Zheng Manqing (1901 1975), Guo Lianying, Shi Diaomei y Xiong Yanghe.

Después de establecerse en la ciudad de Yilan en 1953, Xiong enseñó taiji sin descanso. Finalmente, los practicantes del estilo Xiong llegaron a sobrepasar los 10.000. La contribución más importante de Xiong al legado del taiji es la conservación y transmisión minuciosa del taijiquan Yang como un arte de combate y sistema de ejercicio, siendo lo más notable la práctica por parejas del sanshou. Además, sus libros dejan un detallado registro del sistema.

Incluso en el ocaso de su vida, Xiong se levantaba a las 4:30 de la mañana para comenzar el día, que incluía sus prácticas regulares de taiji. Además del cántico de las escrituras budistas, la práctica de la caligrafía con pincel y la lectura de historia militar, escribió libros que han dejado un registro detallado del sistema taiji para las siguientes generaciones. Era un budista que trató a sus discípulos con un afecto paternal. Murió el 29 de octubre de 1981 en el hospital Yuan Shan Rongmin de Yilan, a la edad de 94 años.

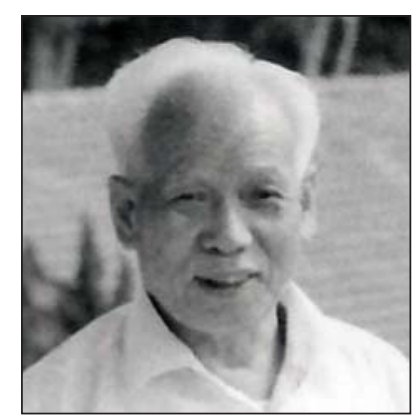

EL MAESTRO YANG QINGYU

(1915 2002) ERA UNO

DE LOS ESTUDIANTES MÁS

VETERANOS DE XIONG

YANGHE Y SU MÁS CERCANO

CONFIDENTE. NACIDO EN

HENAN, SIRVIÓ EN EL EJÉRCITO

Y SE TRASLADÓ A TAIWÁN.

Fotografía de M. DeMarco. 


\section{El currículo de Xiong Yanghe presentado por Lin Jianhong}

En el sitio web Neijia Formosa, David Chesser escribe esto respecto al currículo de Xiong: "Esta cantidad de entrenamiento lo convierte en la versión más completa de taiji practicada en la isla. Simplemente, no he encontrado nada que se le compare"15. Con el objeto de presentar algo del sistema de Xiong Yanghe en esta revista, las fotografías fueron proporcionadas por Robert Yu, que se puso en contacto con el maestro Lin Jianhong durante una visita a Taiwán en octubre de 2006.

El maestro Lin Jianhong estudió con Guo Tingxian, uno de los mejores discípulos de Xiong Yanghe. A través de Guo, Lin también aprendió las Travesuras de los Cinco Animales de Hua Tuo. Lin da clases en el área de Taipei, incluyendo la Plaza de la Libertad (antiguamente llamada la Plaza Conmemorativa de Chiang Kai-shek). El Sr. Robert Yu visitó las clases del maestro Lin tres veces, diciendo que daba gusto conocer a personas como Lin y sus estudiantes, gente "abierta, simpática y hábil" (R. Yu, comunicación personal, 3 de noviembre de 2006). Yu proporcionó más de 100 fotografías y materiales de referencia para este artículo. Ahora, a sus más de cincuenta años, el maestro Lin disfruta enseñando, normalmente con la ayuda de su asistente la Sra. Ye Jinxiu, como se muestra en las siguientes páginas.

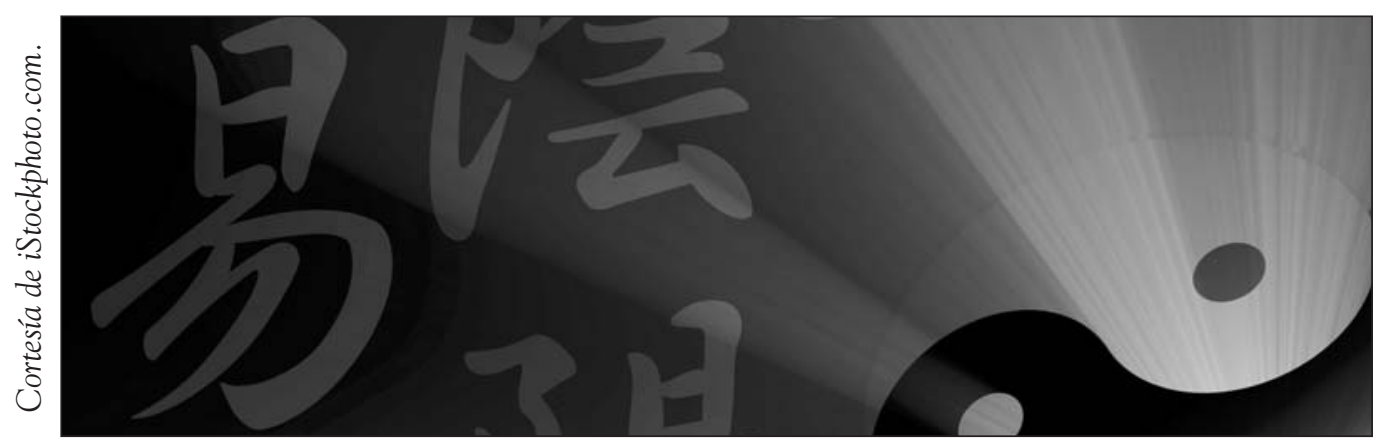

\section{Curriculum del estilo Xiong}

- Taijiquan estilo Xiong de la vieja estructura de la familia Yang (estilo de III)

- Poste básico taiji (de pie)

- Taiji qigong

- Empuje de manos (tuishou)

- Manos dispersantes (sanshou)

- Espada taiji

- Cuchillo

- Bastón

- Palo

- Espadas emparejadas (dos personas)

- Cuchillos emparejados (dos personas)

- Bastones emparejados (dos personas)

- Palos emparejados (dos personas)

- Lanza de la flor de las seis direcciones (Liulu Huaqiang)

- Sable de primavera y otoño

- Espadas dobles

- Boxeo Mizong

- Boxeo Hong de las cuatro puertas

- Boxeo Hong joven

- Palma de la luz solar (Ziyang zhang deng)

- y más
楊家老架熊氏太極拳

太極基本椿

太極氣功

推手

散手

太極劍

刀

棍

桿

對劍

對刀

對桿

對棍

六路花槍

春秋大刀

雙劍

秘宗拳〔迷蹤拳〕

四門洪拳

小洪拳

曦陽掌等 
Why

an. 5 sit Q $x$ xy $\rightarrow 2$

$$
\text { the }
$$
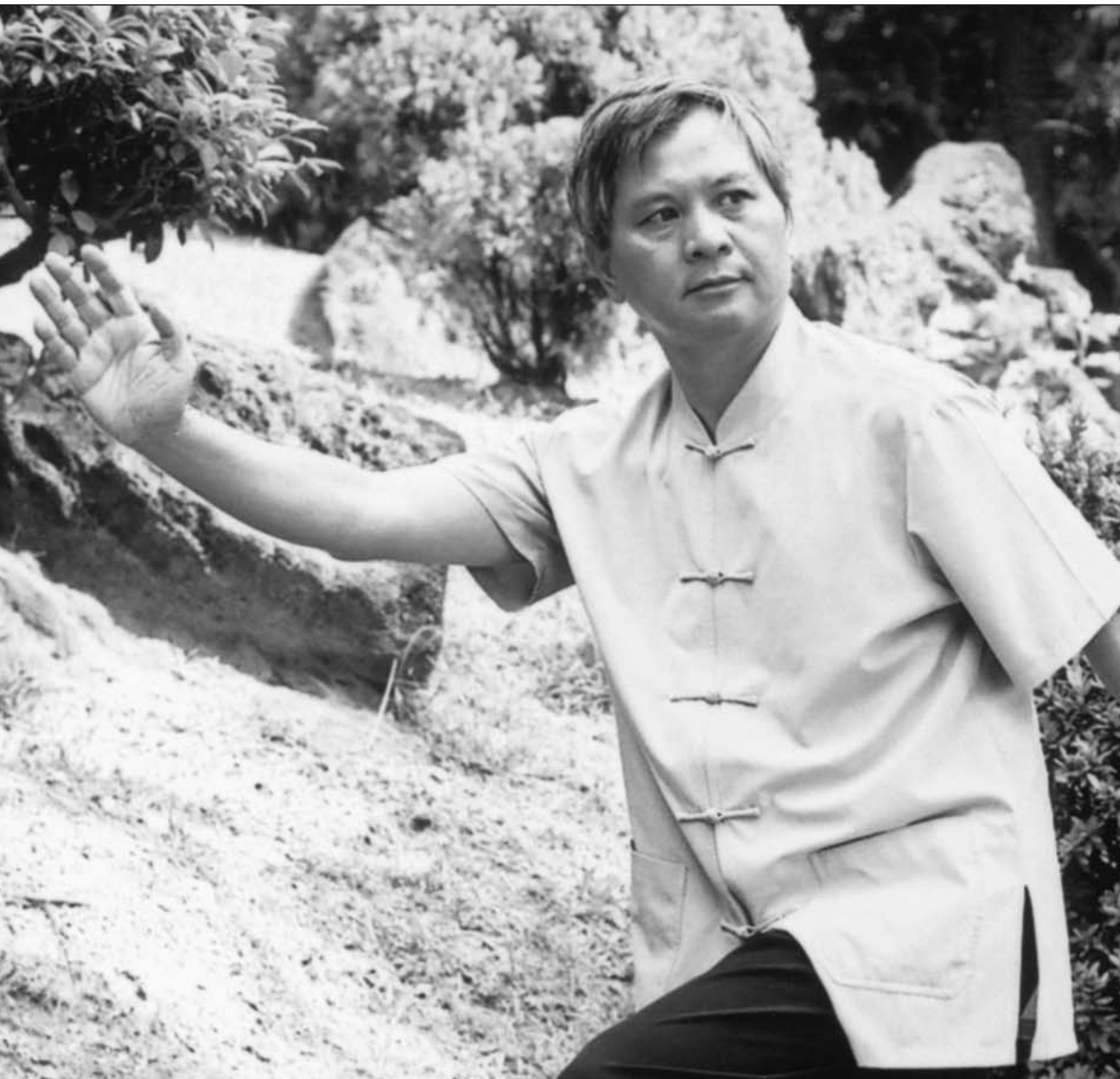


\section{Rutina individual de la forma larga}

POSTURAS SELECCIONADAS DE LA RUTINA TRADICIONAL LARGA, QUE CONSTA DE 111

POSTURAS.
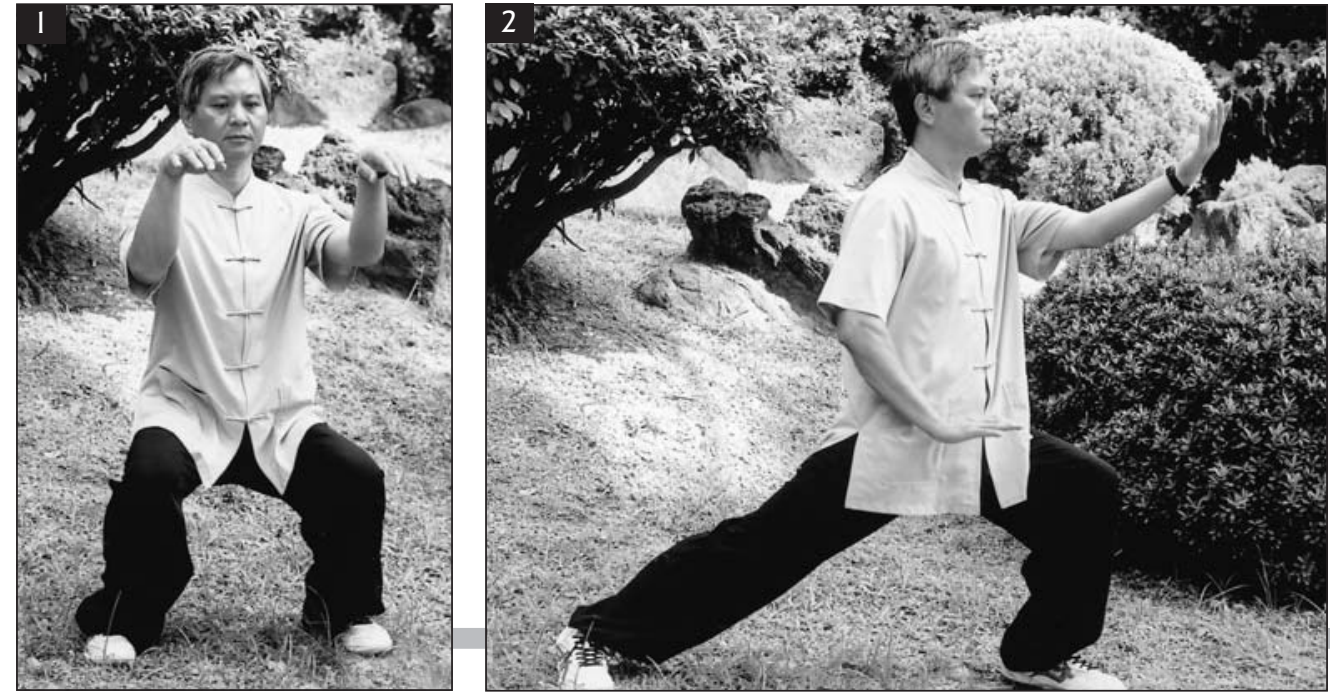

1) Postura de inicio

2) Desvío a la izquierda

3) Desvío a la derecha

4) Enrollar

5) Presionar

6) Codo doble

7) Látigo simple

8) Elevar las manos

9) El gallo se levanta sobre una pata

10) La serpiente se desliza

11) Montar al tigre

12) Tensar el arco Disparar al Tigre
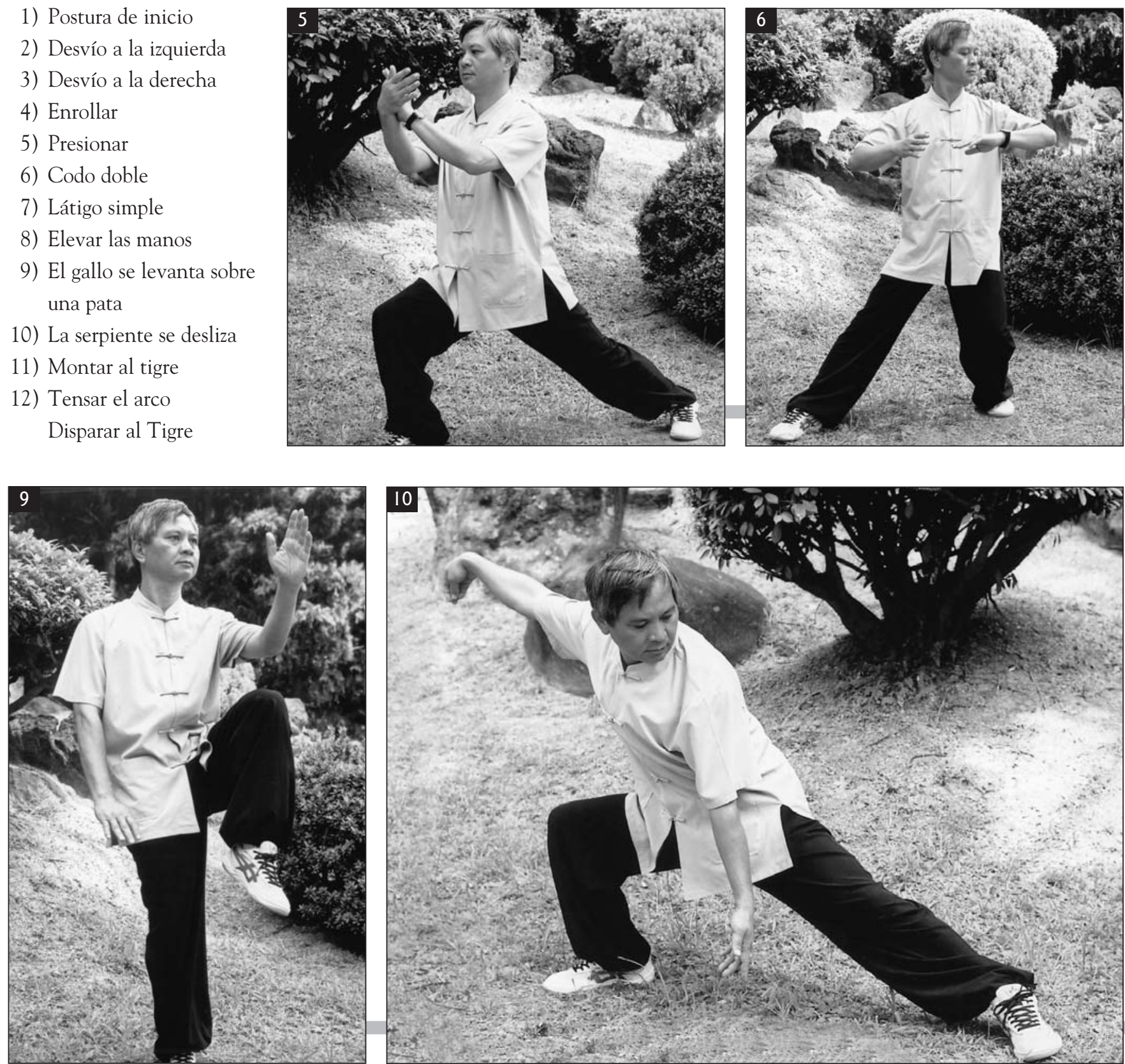

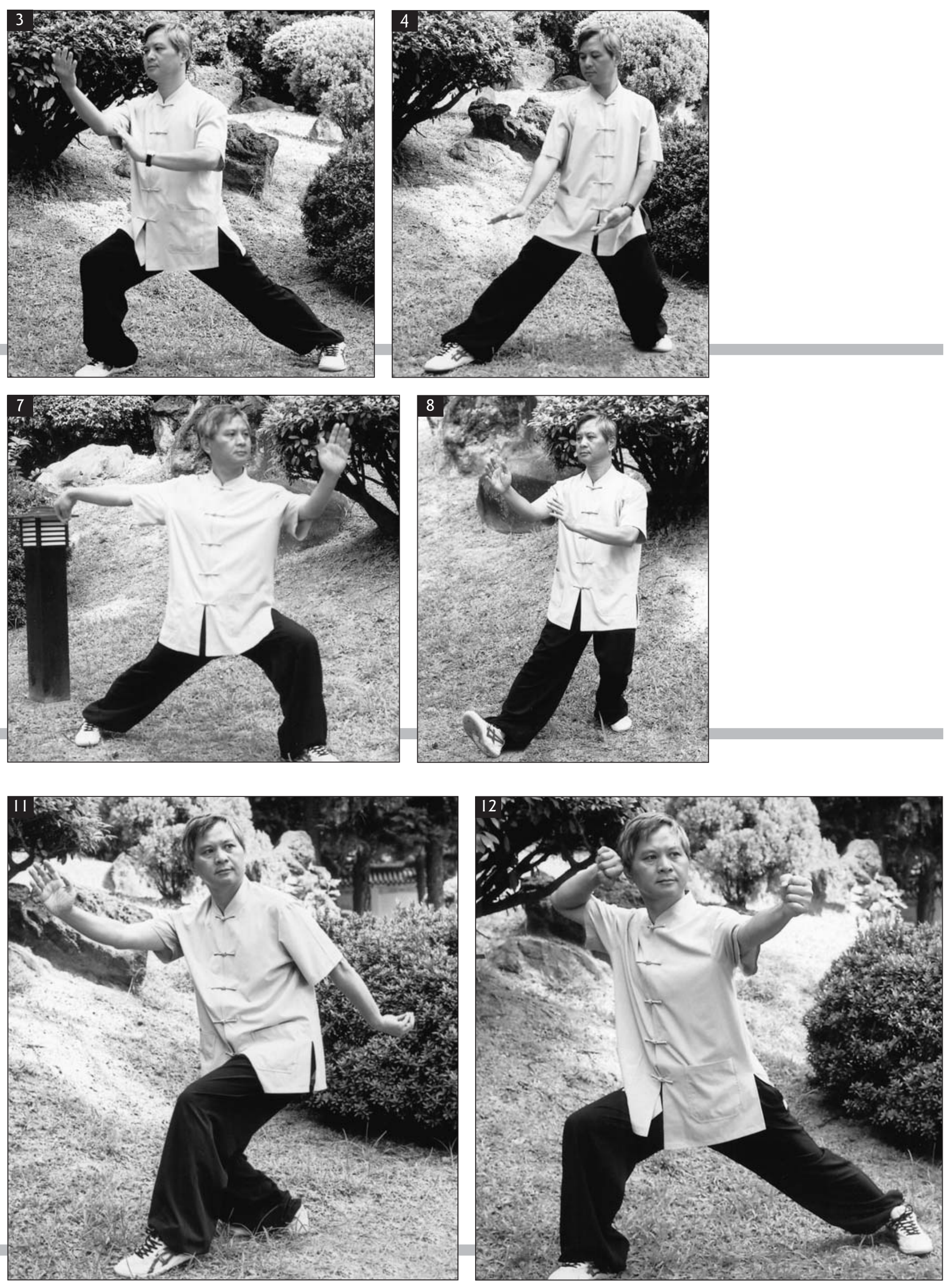

Revista de Artes Marciales Asiáticas $\diamond$ Volumen 4 Número 4 (18-39) - 2009 


\section{Juegos de los cinco animales}

UNA FORMA EXCELENTE DE CULTIVAR LOS PRINCIPIOS DEL TAIJIQUAN ES DEDICAR UNOS MINUTOS AL DÍA A REALIZAR ESTOS EJERCICIOS.

EL MAESTRO LIN TUVO LA FORTUNA DE APRENDER ESTE SISTEMA DE GUO TINGXIAN. LA ESTUDIANTE DE LIN, SRA. YE JINXIU, CONDUCE AL GRUPO.

EL MAESTRO LIN ENSEÑA A DIVERSOS ESTUDIANTES EN DISTINTOS LUGARES Y A DIFERENTES HORARIOS. LA MAYORÍA QUIERE APRENDER TAIJI POR SALUD Y COMPAÑERISMO. OTROS, NORMALMENTE LOS ESTUDIANTES MÁS JÓVENES, PROFUNDIZAN EN LAS TRADICIONES DE COMBATE. A PESAR DE TODO, SI ESTUdiAS EL ESTILO XIONG, CONSEGUIRÁS UNA MEZCLA DE AMBAS DEBIDO A LO COMPLETO DE ESTE SISTEMA TRADICIONAL.

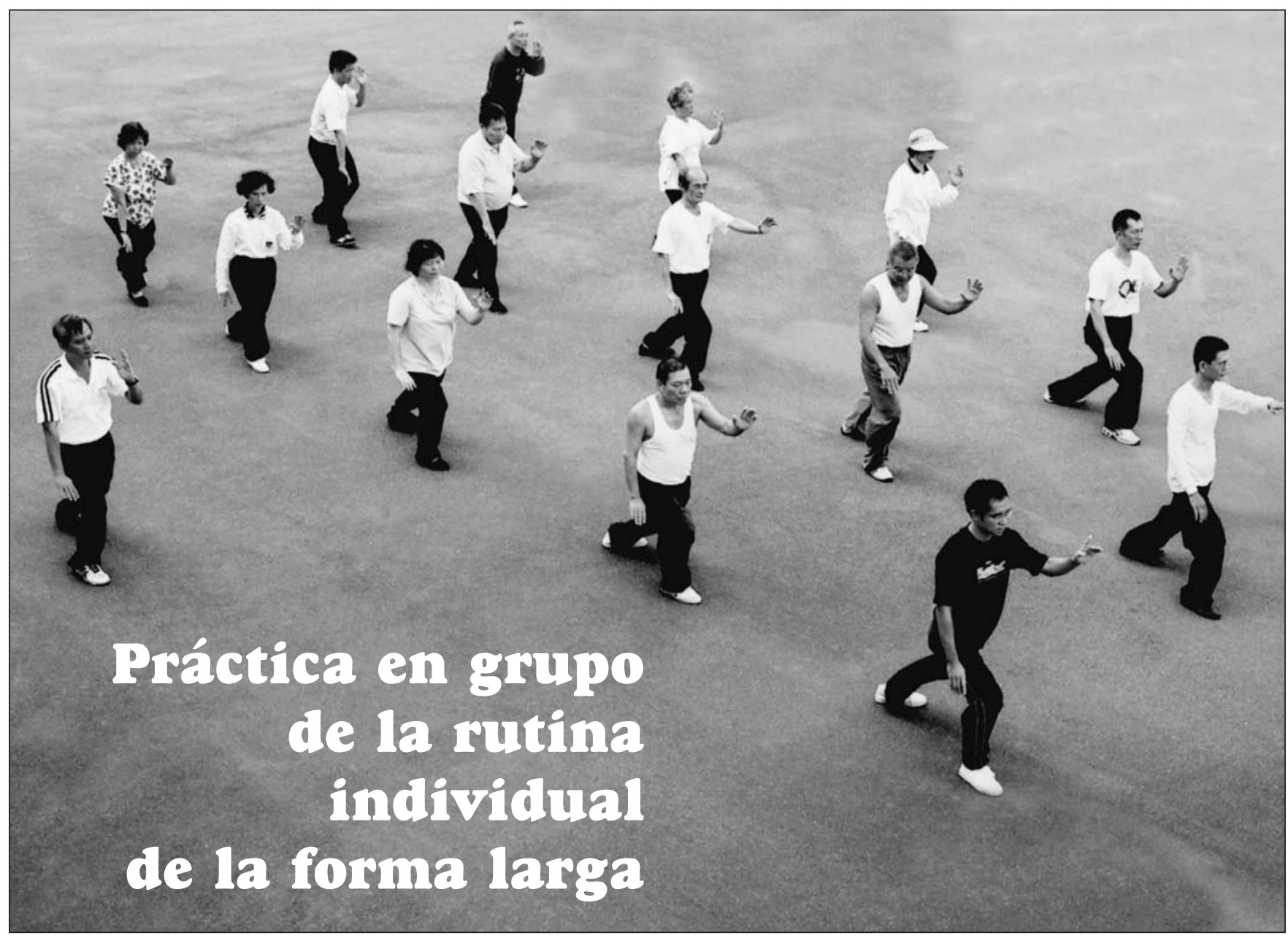




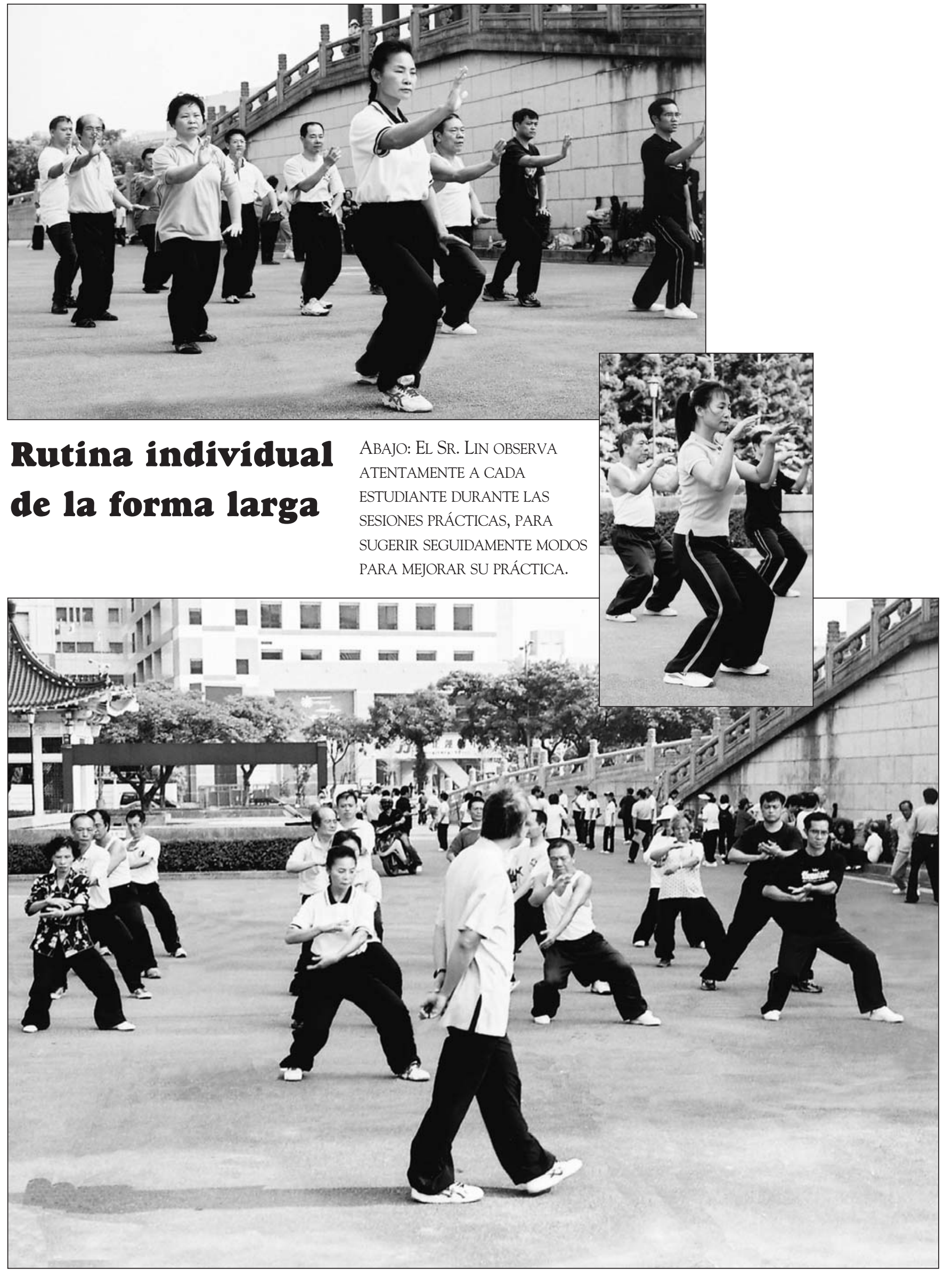



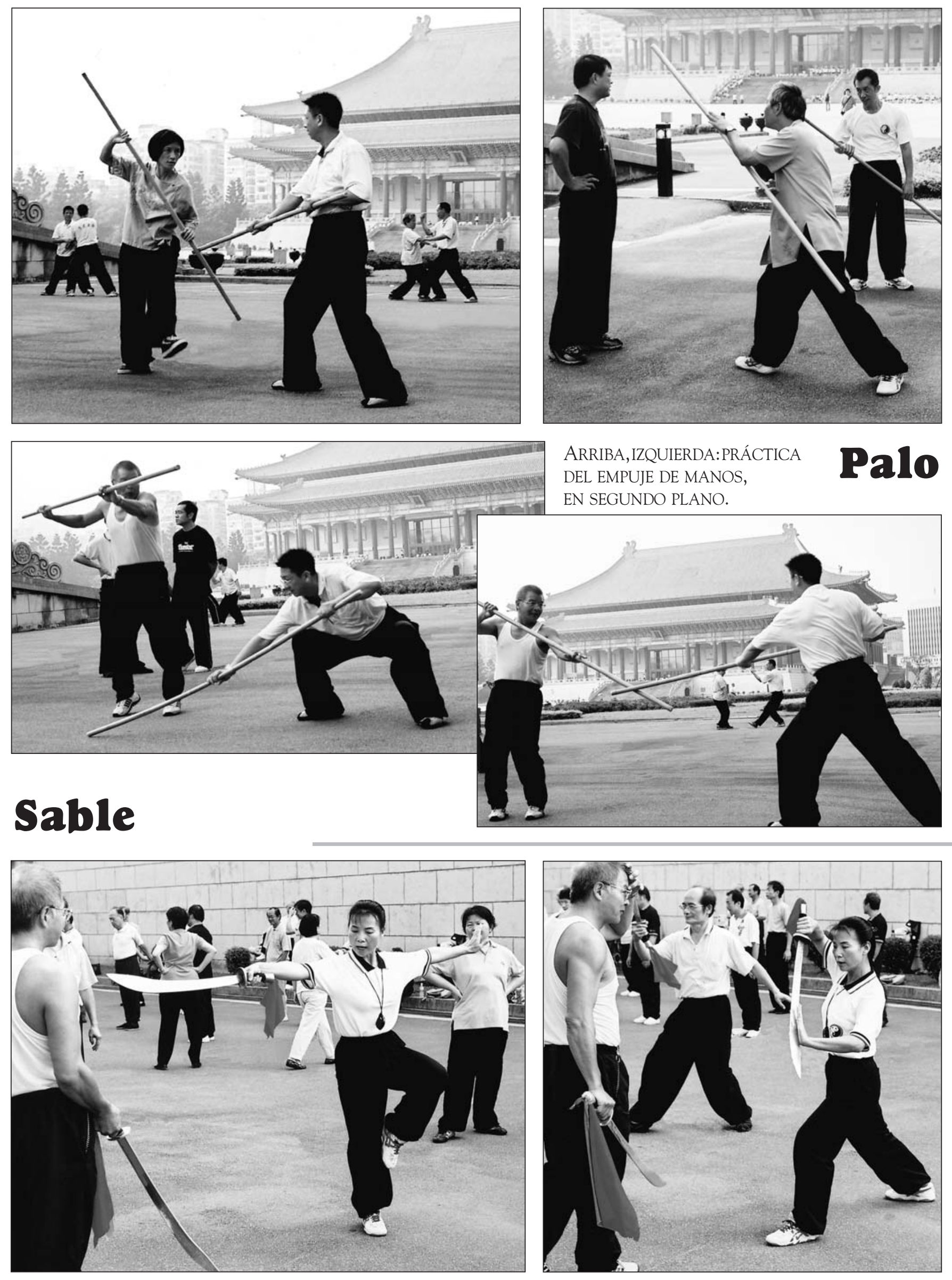
Arriba,izQuiErda:práctica
Del empuje de manos, EN SEGUNDO PLANO.
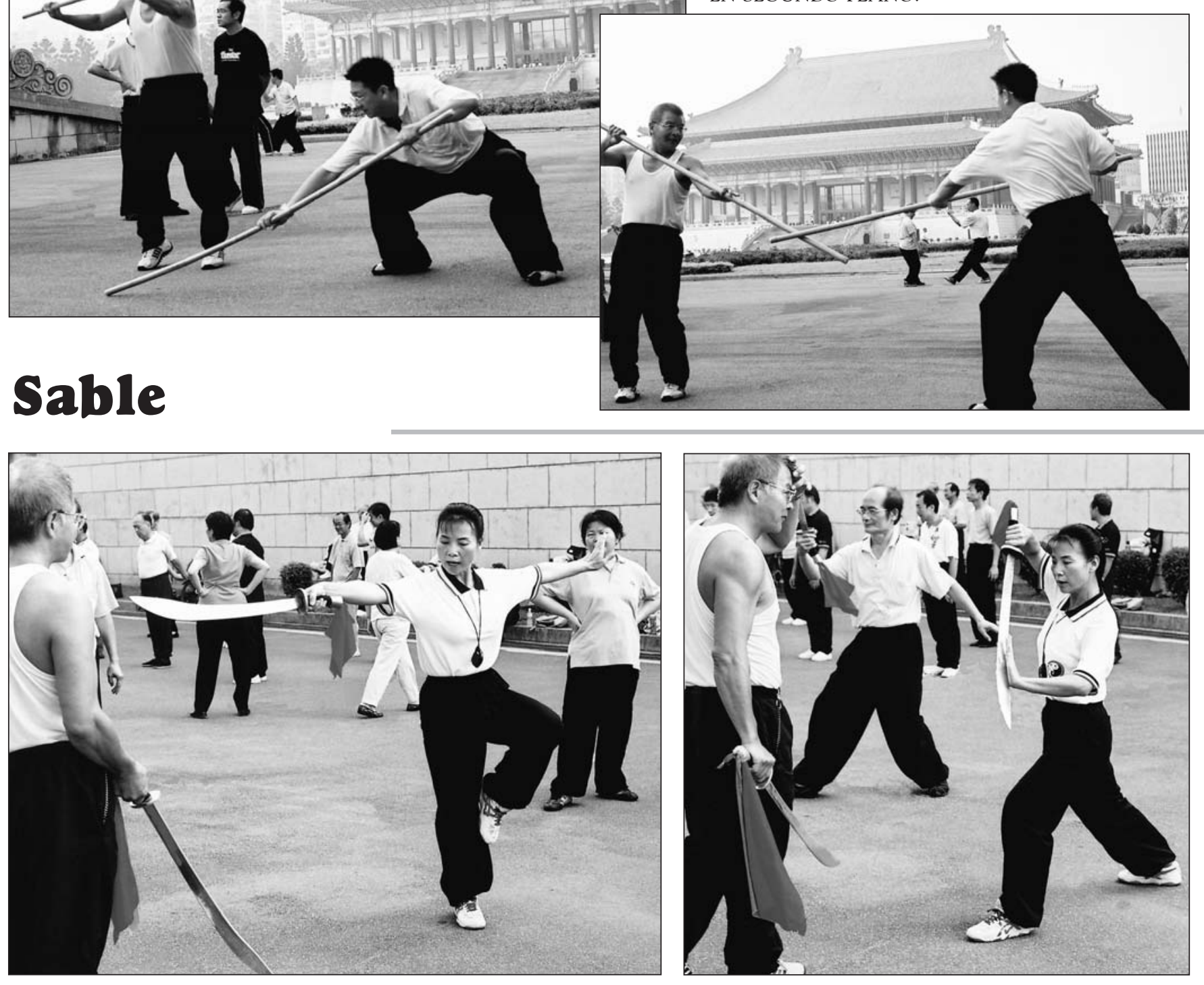

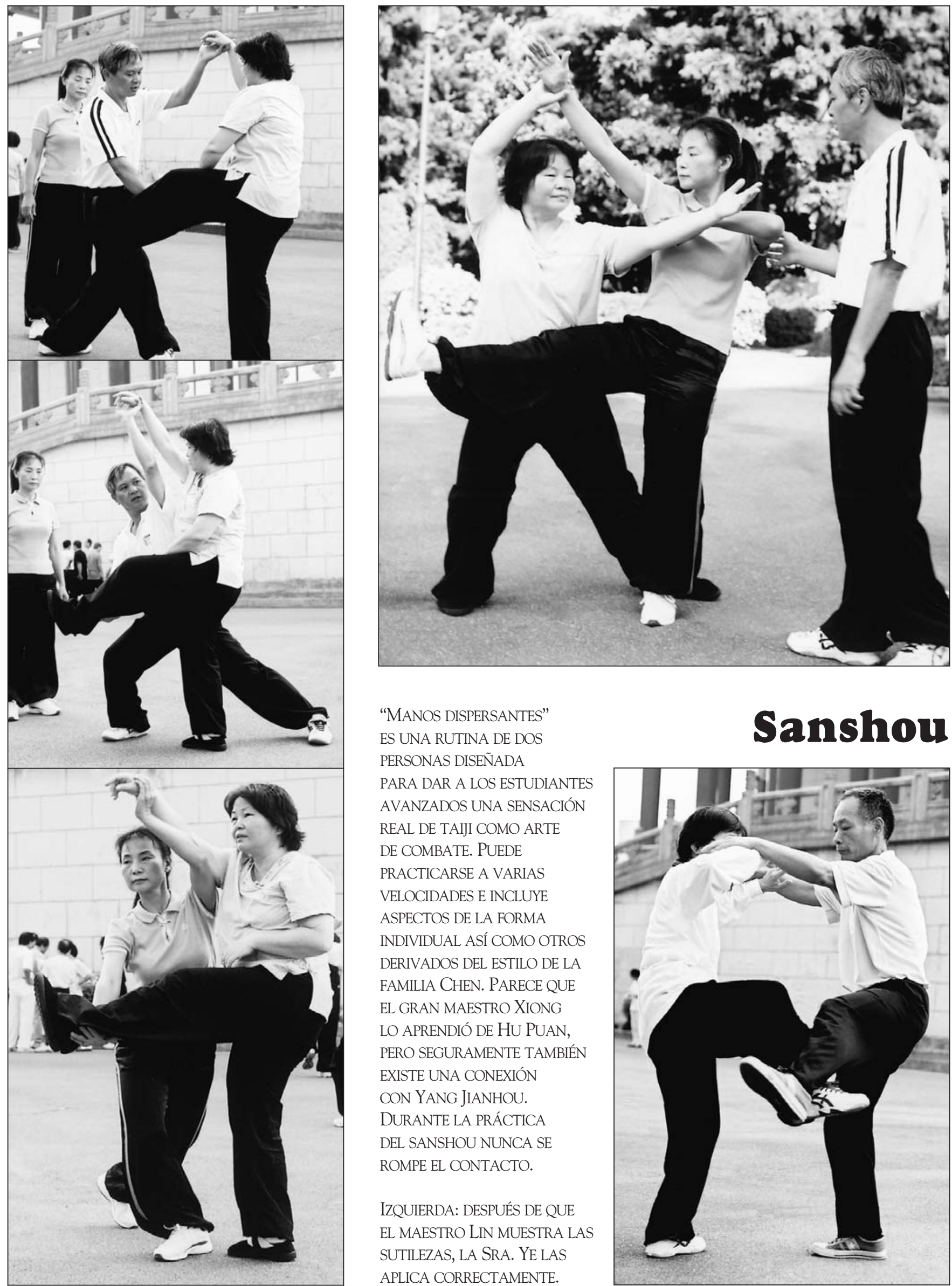

"MANOS DISPERSANTES" ES UNA RUTINA DE DOS Sanshou PERSONAS DISEÑADA PARA DAR A LOS ESTUDIANTES AVANZADOS UNA SENSACIÓN REAL DE TAIJI COMO ARTE DE COMBATE. PUEDE PRACTICARSE A VARIAS VELOCIDADES E INCLUYE ASPECTOS DE LA FORMA INDIVIDUAL ASÍ COMO OTROS DERIVADOS DEL ESTILO DE LA FAMILIA CHEN. PARECE QUE EL GRAN MAESTRO XIONG LO APRENDIÓ DE HU PUAN, PERO SEGURAMENTE TAMBIÉN EXISTE UNA CONEXIÓN CON YANG JIANHOU.

DURANTE LA PRÁCTICA DEL SANSHOU NUNCA SE ROMPE EL CONTACTO.

IZQUIERDA: DESPUÉS DE QUE EL MAESTRO LIN MUESTRA LAS SUTILEZAS, LA SRA. YE LAS APLICA CORRECTAMENTE.

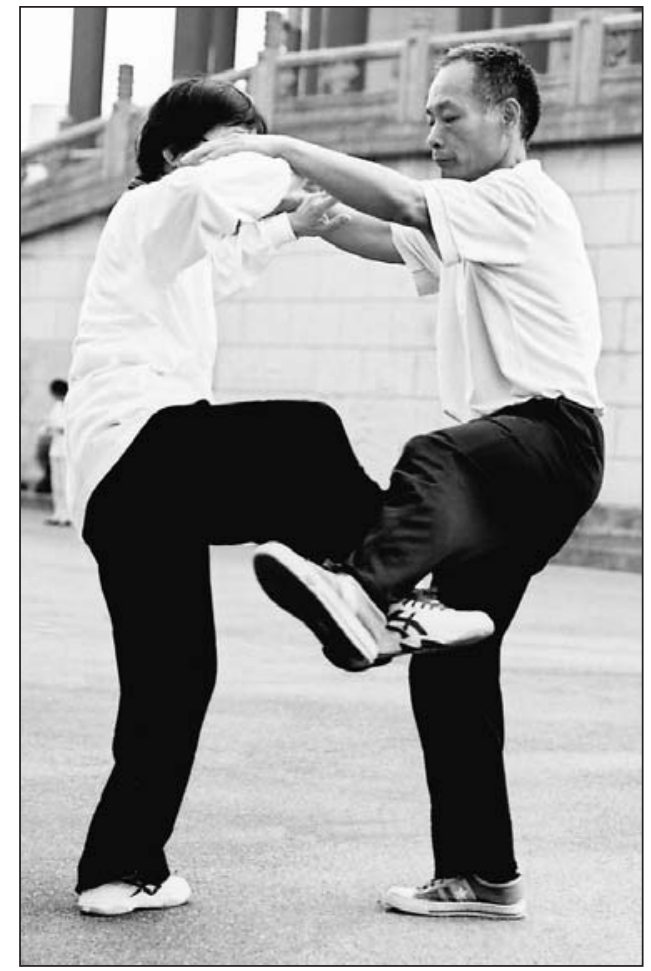




\section{Comentarios finales sobre el taijiquan estilo Xiong}

Como la historia de los ciegos que examinaban a un elefante, este artículo sólo puede representar los resultados que ha encontrado el autor, que son limitados debido a una relativa falta de materiales de referencia, la dificultad en la exactitud de la traducción de los textos chinos y tiempo disponible para la investigación. Asumo la responsabilidad de cualquier defecto y agradezco cualquier feedback útil. Con un poco de suerte, a pesar de estas limitaciones el material que aquí se ha presentado puede ampliar la perspectiva sobre el taijiquan, considerando el marco histórico donde los principales representantes del linaje de la familia Yang desarrollaron el arte.

Hemos encontrado que hubo dos factores principales que influyeron en el desarrollo inicial del estilo Yang. El primero es la casi incomprensible violencia que existió desde la caída de la dinastía Qing hasta la fundación de la República Popular China, especialmente durante el Periodo Republicano (1911 1949), que empapó la tierra china de sangre cuando el desorden estaba en su cénit. Fue una época en la que muchos buscaron métodos de combate superiores y los practicaron como si sus vidas dependieran de ellos. No es sorprendente por tanto que el taijiquan fuese un sistema que se desease aprender y que emigrase desde un pequeño pueblo para ser practicado por guardaespaldas en las ciudades más grandes donde se encontraba el personal militar y policial.

El otro factor principal que influyó sobre la historia del taiji brota del agotamiento sentido por el país y su gente tras siglos de rebeliones e intervenciones extranjeras. Años de combate, derrota y humillación inspiraron en el país un sentimiento creciente de nacionalismo y una era de "auto-fortalecimiento". Una forma de curar al "hombre enfermo de Asia" fue extender el taiji para la salud: se encontró que era muy efectivo como forma de ejercicio, no se necesitaba una ropa o un lugar especial, y era económico cuando se practicaba en grupos. Millones de personas están más sanas gracias a él.

Si tenemos presentes estas dos influencias mientras observamos los inicios del estilo Yang, se revela una interrelación especial entre el taiji y la historia social china. Entre el nacimiento de Yang Luchan y la muerte de Xiong Yanghe, las facciones del pueblo chino lucharon por sobrevivir durante 150 años antes de que finalmente emergiera como una nación en paz. Sin duda, el taiji de Yang Luchan era un arte de combate, pero ¿a qué se parecía? ¿cómo lo practicaba? ¿cuál era la profundidad de su conocimiento?

La mayoría de estilos de taiji actuales han evolucionado desde las mismas raíces marciales. Esta evolución corre paralela al descenso de la violencia en China y el crecimiento de la estabilidad social y política. En sus niveles más altos, el taiji como arte de combate siempre se ha transmitido a un número relativamente pequeño de personas. La enseñanza en masa para la salud pública ha alcanzado a millones. Como resultado, una inmensa mayoría de practicantes de taiji conocen la forma, pero poco de la función. Las razones que uno tiene para aprender taiji afectan a cómo se practica y observa la forma. No podemos ver cómo practicaba Yang Luchan, pero el sistema conservado por Xiong Yanghe parece ser un buen indicador y es valorado por preservar una gran tradición en Taiwán que estuvo casi perdida durante la Revolución Cultural Comunista (1966 1976), un movimiento social que incluyó una cruzada para despojar a China de las "formas antiguas de pensar", tales como aquellas que se mostraban en las artes marciales tradicionales.

El estilo Xiong ofrece elementos de combate que fueron necesarios durante el caos extremo en que se encontró China durante las primeras transmisiones del arte de la familia Yang. Yang Luchan estudió el estilo Chen y aspectos de este estilo también se han reflejado en el estilo Xiong: las posturas son a menudo bajas y amplias, las aplicaciones son efectivas, los métodos de entrenamiento en el empuje de manos y en el sanshou son prácticos y comprenden la utilización de armas. Aunque el sistema de Xiong retiene el viejo toque Yang, Xiong vivió cuarenta años más que Yang Chengfu, y dentro de la época post-1949. Dos razones le motivaron para dar clases. Enseñó a sus estudiantes cercanos el taiji como un arte de combate y como un ejercicio para la salud y la longevidad. Enseñó taiji a otros miles básicamente para el "auto-fortalecimiento".

Taiji Estilo Xiong en Taiwán $\diamond$ Michael A. DeMarco 
Esta breve perspectiva del estilo Xiong ayuda a definir y dar sentido a las palabras "taijiquan". El taiji no es sólo un ejercicio ni un arte de combate. Es ambos, y su naturaleza dual es inherente a las enseñanzas de los verdaderos maestros. Alguien que domina el estilo Xiong, o los primeros sistemas de la familia Yang, puede impartir la teoría y el conocimiento aplicable en ambas áreas, como arte de combate y sistema de ejercicio. La mezcla está determinada en gran medida por la relación profesor-estudiante y las motivaciones que entran en juego. El taijiquan del estilo Xiong de la familia Yang nos da una oportunidad única para mirar atrás en el tiempo cuando se forjó la plantilla del estilo Yang. A medida que el sistema prospera en Taiwán gracias a los discípulos de Xiong y a los discípulos de sus discípulos - profesores como Lin Jianhong y Lin Chaolai- vemos que el viejo sistema ha sido conservado, a la vez que se ha extendido fuera de Taiwán para beneficiar a otros. Es el sabor de un "viejo vino en una botella nueva".

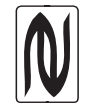

\section{AGRADECIMIENTOS}

El ánimo para escribir este artículo vino del Sr. Robert Lin-I Yu, un destacado instructor de baguazhang y xingyi (discípulo de Hong Yixiang, Zhou Jincai y otros en Taiwán). Desde su casa en Madison, Wisconsin, Yu llamó para decir que regresaría a Taiwán en octubre (2006) para dirigir una investigación de artes marciales y visitar a los parientes. "¿Quieres que haga algo por ti mientras estoy allí?”. Mi única respuesta fue que, si quería, podía contactar con el Sr. Lin Shengxuan (uno de mis compañeros de clase de taiji del estilo Xiong) y cualquier otro instructor del estilo Xiong que pudiera encontrar. Investigó y regreso a los EE.UU. con un gran montón de fotografías, notas, copias de manuscritos, libros y manuscritos inéditos de los instructores del estilo Xiong. Un agradecimiento especial a estos dos excelentes caballeros por su amistad y apoyo, y al maestro Lin Jianghong, la Sra. Ye Jinxiu y a los demás compañeros de clase por colaborar tan amablemente.

\section{REFERENCIAS DE FUENTES DE SITIOS WEB}

${ }^{1}$ http://yuehuanzhi.blog.sohu.com

${ }^{2}$ www.taiji.net.cn/liu/wlys/200712/6426.shtml; http://yuehuanzhi.blog.sohu.com

${ }^{3}$ http://blog.udn.com/article/trackback.jsp?uid=wang6196192001\&aid=1077817

${ }^{4}$ http://tw.myblog.yahoo.com/q3taichi/article?mid=23\&sc $=1$

${ }^{5}$ http://library.taiwanschoolnet.org; http://blog.youthwant.com.tw/vadjra/vadjra/6395839/

${ }^{6}$ http://yuehuanzhi.blog.sohu.com; www.xici.net/u6819319/d19792891.htm

${ }^{7}$ http://tw.myblog.yahoo.com/q3taichi/profile

${ }^{8}$ www.xici.net/u6819319/d19792891.htm

${ }^{9}$ www.dotaichi.com

${ }^{10} \mathrm{http} / / /$ blog.sina.com.tw/lkk_blog/article.php?pbgid=36074\&entryid=320007

${ }^{11} \mathrm{http}: / /$ www.lin-gi.com.tw/discuss/Viewtopic.asp?Subject $\mathrm{D}=7135 \&$ Sign=150

${ }^{12} \mathrm{http}: / /$ tw.myblog.yahoo.com/jin_cang/article? $\mathrm{mid}=1003 \& \mathrm{prev}=2170 \&$ next $=554 \&$ $=f \& f i d=3$

${ }^{13}$ http://blog.udn.com/wang6196192001/1067085

${ }^{14} \mathrm{http}: / /$ taiwanreview. nat.gov.tw/fp.asp?xItem $=589 \& \mathrm{CtNode}=128$

${ }^{15} \mathrm{http}: / /$ chessman71.wordpress.com/2006/05/15/yang-shao-hous-taiji/) 


\section{FOTOGRAFÍAS}

\section{DE XIONG YANGHE}

Como parte de su aspiración de conservar los registros culturales, el Programa Nacional de Archivos Digitales de Taiwán (véase www.ndap.org.tw) tiene fotografías digitales de Xiong Yanghe en su colección que pueden verse como miniaturas y en formato normal

(véase

http:// digitalarchives.tw).

\section{REFERENCIAS CHINO}

ANÓNIMO, (1987). Mr. Xiong's 100th birthday commemorative special edition. (s.l.).

ANÓNIMO, (1984). National arts master Xiong Yanghe commemorative collection. (s.l.).

LIN, CAOLAI (2007). Yang family old frame Xiong style taijiquan. DVD. Yilan, Taiwan: Chin-yu Martial Art Study Association.

YANG, QINGYU (1976). Xiong style taijiquan long form, push-hands, and sword form. Colección cinematográfica privada.

YANG, QINGYU (1988). Autobiography. Auto-publicada.

YANG, QINGYU (n.d.). A brief biography of Xiong Yanghe. Auto-publicada.

XIONG, Y.H. (1962). Autobiography. Auto-publicada.

XIONG, Y.H. (1963). The taijiquan explained. Taipei: Taiwan China Book Printing House.

XIONG, Y.H. (1971). Taiji swordsmanship illustrated. Yilan, Taiwan: Lu Feng Printing and Publishing House.

XIONG, Y.H. (1975). The taijiquan explained. $3^{\mathrm{a}}$ edición. Taipei: Huge Distribution Planning Company.

\section{REFERENCIAS INGLÉS}

HUCKER, C. (1975). China's imperial past: An introduction to Chinese history and culture. Stanford, CA: Stanford University Press.

DeMArCo, M. (1992). The origin and evolution of taijiquan. Journal of Asian Martial Arts, $1(1): 8-25$.

GallaghER, P. (2007). Drawing silk: Masters' secrets for successful tai chi practice. Charleston, SC: BookSurge.

HAYWARD, R. (2000). T'ai-chi ch'uan: Lessons with master T.T. Liang. St. Paul, MN: ShuKuang Press.

KuHN, P. (1970). Rebellion and Its Enemies in Late Imperial China: Militarization and Social Structure, 1796 1864. Cambridge, MA: Harvard University Press.

KURland, H. (May 1998). "Hsiung Yang-Ho's san shou form." T'ai chi Ch'uan and Wellness Newsletter. Descargada el 16 de Julio de 2009.

KuRland, H. (2003). "History of a rare t'ai-chi form: San shou." http://www.selfgrowth. com/articles/Kurland3.html. Descargada el 16 de Julio de 2009.

LU, S. (Yun, Z., Trans.) (2006). Combat techniques of taiji, xingyi, and bagua. Berkeley, CA: Blue Snake Books.

Olson, S. (1999). T'ai chi thirteen sword: A sword master's manual. Burbank, CA: MultiMedia Books.

Olson, S. (1999). T'ai chi sensing-hands: A complete guide to t'ai chi t'ui-shou training from original Yang Family records. Burbank, CA: Multi-Media Books.

Olson, S. (1992). The teachings of master T.T. Liang: Imagination becomes reality, the complete guide to the 150 posture solo form. St. Paul, MN: Dragon Door Publications.

Russell, J. (2004). The tai chi two-person dance: Tai chi with a partner. Berkeley, CA: North Atlantic Books.

SHERIDAN, J. (1977). China in Disintegration: The Republican Era in Chinese history 1912 1949. New York: The Free Press.

SChurmann, F. \& SChell, O. (1967). Republican China: Nationalism, war, and the rise of Communism 1911-1949. New York: Vintage Book.

Wakeman, F. (1977). The fall of imperial China. New York: The Free Press.

Wile, D. (1996). T'ai-chi touchstones: Yang family secret transmissions. Brooklyn, NY: Sweet Chi Press.

WILE, D. (1996). T'ai-chi touchstones: Yang family secret transmissions. Brooklyn, NY: Sweet Chi Press. 


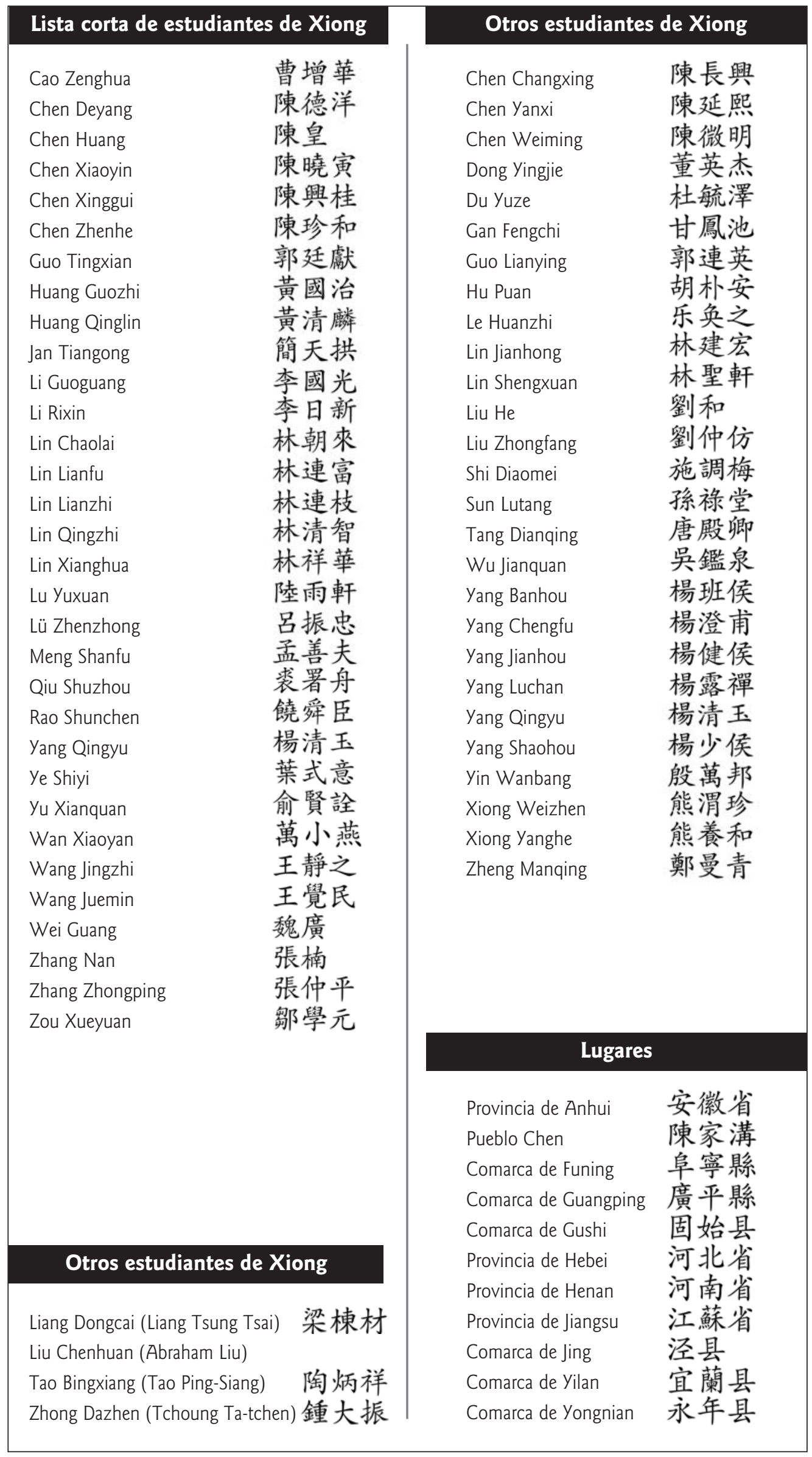

